\title{
Real-time parallel implementation of Pulse-Doppler radar signal processing chain on a massively parallel machine based on multi-core DSP and Serial RapidlO interconnect
}

\author{
Abdessamad Klilou ${ }^{1 *}$, Said Belkouch¹, Philippe Elleaume ${ }^{2}$, Philippe Le Gall², François Bourzeix ${ }^{3}$ \\ and Moha M'Rabet Hassani ${ }^{1}$
}

\begin{abstract}
Pulse-Doppler radars require high-computing power. A massively parallel machine has been developed in this paper to implement a Pulse-Doppler radar signal processing chain in real-time fashion. The proposed machine consists of two C6678 digital signal processors (DSPs), each with eight DSP cores, interconnected with Serial RapidIO (SRIO) bus. In this study, each individual core is considered as the basic processing element; hence, the proposed parallel machine contains 16 processing elements. A straightforward model has been adopted to distribute the Pulse-Doppler radar signal processing chain. This model provides low latency, but communication inefficiency limits system performance. This paper proposes several optimizations that greatly reduce the inter-processor communication in a straightforward model and improves the parallel efficiency of the system. A use case of the Pulse-Doppler radar signal processing chain has been used to illustrate and validate the concept of the proposed mapping model. Experimental results show that the parallel efficiency of the proposed parallel machine is about $90 \%$.
\end{abstract}

Keywords: Parallel computing; Real-time processing; Pulse-Doppler radar; Digital signal processor; Serial RapidIO interconnect

\section{Introduction}

Radar is an object detection system. It transmits pulses of radio waves or microwaves which bounce off any object in their path, and based on the received echo, range, altitude, direction, or speed of objects can be determined.

Radar systems can be classified into two main categories depending on the used technology: primary radars or secondary radars. Primary radar transmits high-frequency signals which are reflected by the target and then received by the same radar. The reflected echoes will be processed to extract target information. Secondary radar, in addition to primary radar characteristics, uses a transponder on the target for responding to interrogation by transmitting a coded reply signal. We can distinguish between different techniques in primary radars. Continuous wave primary

\footnotetext{
* Correspondence: a.klilou@ced.uca.ma

${ }^{1}$ Ecole Nationale des Sciences Appliquees - Marrakech, University of Cadi Ayyad, Marrakech, Morocco

Full list of author information is available at the end of the article
}

radar transmits and receives continuously high-frequency signals, and a spatial separation between transmission and reception must be present. Pulse-Doppler radars transmit short pulses at a certain repetition frequency, and the receiver and the transmitter share the antenna using a duplexer [1].

The radar studied in this paper is Pulse-Doppler radar containing a network of uniform linear antenna and using beamforming to control the field of view of the radar. The signal processing chain applied to the received echo requires high-computing power to extract target information in real time [2]. A massively parallel machine with multiple processing elements that operate concurrently has been proposed in this paper to implement the totality of the signal processing chain in real-time fashion.

The parallel machine presented in this paper consists of a multi-core DSP as a basic processing element and SRIO as an inter-processor communication bus. We studied the distribution of the Pulse-Doppler radar signal processing 
chain algorithm on the proposed parallel machine. We used a straightforward model to distribute the processing on the parallel machine. The straightforward partitioning method provides low latency but generate communication inefficiency associated with the large communication group [3]. This limits the parallel efficiency of the system. The goal of this paper is to propose optimizations that reduce the inter-processor communication in the straightforward model and improves the parallel efficiency of the system.

We have chosen the multi-core C6678 DSP, as the basic processing element, for its low power consumption and high-performance fixed/floating point calculations [4]. C6678 DSP is an eight-core device; each core can run up to $1.25 \mathrm{GHz}$. It provides a peak performance of 160 GFLOP for a floating point and 320 GMAC for a fixed point for only 10 watts. It has been used recently by many research communities to build high-performance and low power real-time signal processing systems [2,5-11]. Additionally, SRIO is integrated in the C6678 DSP as a peripheral device.

To have good performances in a multi-processor environment, processors must communicate with an efficient inter-processor communication bus. This communication bus must belong to the processor-to-processor category and not to the processor-to-IO category like USB, PCI, or PCIe; it must have a high data rate and a good efficiency. Following our previous research works [12], the SRIO protocol is proposed to interconnect between DSPs in the proposed parallel machine. SRIO is a high-performance, packet-switched, interconnect technology. It was developed to address the embedded industry's need in terms of faster bus speeds; increased bandwidth and reliability in an intra-system interconnect. SRIO allows chip-to-chip and board-to-board communications at performance levels scaling from 1 gigabit per second per link up to 20 Gbps [13].

We proposed an experimental platform with two C6678 DSPs, each with eight DSP cores, interconnected by SRIO, and a use case of the Pulse-Doppler radar signal processing chain to illustrate and validate the proposed optimizations.

The remainder of this paper is organized as follows. Section 2 presents recent research about SRIO and mapping methods of radar algorithms on multi-processor systems, including a brief description of our previous work with SRIO. Section 3 introduces Pulse-Doppler radar. Section 4 describes the experimental platform. We will give an overview of the C6678 DSP, the RapidIO protocol, and the SRIO version implemented in the C6678 DSP. Then, we present the performance evaluation studies of SRIO. In Section 5, experiments and results are presented. We will start by describing the straightforward model used to map the Pulse-Doppler radar processing chain on the parallel machine. Then we will present all optimizations performed to the straightforward model to improve the parallel efficiency of the system. Finally, a conclusion is provided in Section 6.

\section{Related works}

In recent years, many research communities were interested in studying the SRIO interconnect to develop highperformance computing systems.

Adams et al. [14] have done a simulation analysis of a high-performance processing architecture based on RapidIO. Two network topologies were simulated: a simple network consisting of an 8-port switch and 8 processing nodes and a more extensive network consisting of five 8port switches and 24 processing nodes. Results indicate that latencies as low as $92 \mathrm{~ns}$ for a remote 64-bit read request/response transaction may be achieved in an unloaded single-switch system.

$\mathrm{X}$ Zhang et al. [15] were interested in developing highperformance technologies to meet the demanding requirement of future real-time signal processing applications. A universal, flexible, and high-performance signal processing module based on the TMS320C6455 DSP and RapidIO interconnect were developed for a next-generation, scalable, modular, and adaptable signal-processing system such as radar jamming system. X Zhang et al. [16] have also developed two kinds of flexible signal processing modules based on high-performance ADC and heterogeneous processors. They used RapidIO as an interconnect technology. The receiver module mainly consists of two 10bit 2Gsps ADCs, one TMS320C6455 DSP and one XC5V95T fieldprogrammable gate array (FPGA). The processing module is composed of four TMS320C6455 DSPs and one XC4VSX55 FPGA. The constructed system shows the application of a multi-channel receiver, and it is scalable in resources and application.

Changrui et al. [17] have designed a high-performance heterogeneous embedded signal processing system based on the SRIO interconnection for radar systems application. The system consists of three modules which are high-speed AD module, heterogeneous processing module including FPGA and central processing unit (CPU), and RapidIO switch module. The developed system has achieved a high-performance computation with up to $550 \mathrm{MHz}$ on FPGA and up to $1.5 \mathrm{GHz}$ on a CPU. The system is scalable and allows future enhancements.

Y Zhang et al. [18] have developed a flexible and highperformance signal-processing module based on the SRIO interconnect and Advanced Mezzanine Card (AMC) modules to meet the requirements of scalability, adaptability, and computational capability of next-generation signalprocessing systems. A prototype of 12 modules was introduced, and an application of this computing system in a MIMO-OFDM system was covered. They claimed that their system, which also include three kinds of interconnect 
methods (gigabit Ethernet, SRIO, and RocketIO), can be adopted as a hardware platform for radars.

J Zhang et al. [19] have done experiments on SRIO. They have used four C6455 DSPs interconnected pointto-point by SRIO. They have measured the data rate of the communication by SRIO in direct IO mode. Experimental results show that the read and write operation can stably work at 3.125 Gbps per channel between different DSPs. The data rate obtained is up to $275 \mathrm{MB} / \mathrm{s}$ when the baud rate is 3.125 Gbps.

Xue et al. [20] have proposed a new implementation platform for parallel fast Fourier transform (FFT) called multi-core DSPs. They have used SRIO to achieve DSPto-DSP communication and enhanced direct memory access (EDMA) to perform core-to-core communication. The proposed implementation has improved the efficiency of parallel FFT, especially for large points; the speed-up obtained is about 2.95 when the FFT is distributed on four DSP cores.

Our previous research work [12] was addressing the performance optimization of the SRIO interconnect. We have used an experimental test bed composed of two multi-core C6474 DSPs connected by two links of SRIO V1.3. We have measured the data rate of different transactions proposed by SRIO in the Direct IO mode. We have also proposed three methods to transfer a data stream greater than $4 \mathrm{~KB}$. Experimental results show that Nwrite and Swrite transactions achieve more performances than Nwrite_R and Nread transactions. We have determined that in order to transfer a data stream, the interrupt method is the simplest. In addition, it increases transfer robustness. The performance difference between the interrupt method and method of the EDMA in synchronized mode is tolerable.

Bueno et al. [3,21-25] were interested in using the RapidIO embedded interconnect in system architectures for space-based radar. They have studied two algorithms: ground moving target indicator (GMTI) and synthetic aperture radar (SAR). Their work focused on the modeling and the simulation of RapidIO hardware and related applications using MLDesigner [26]. They have developed a RapidIO test bed using Xilinx Virtex II Pro FPGA chips and boards, and a Tundra 4-port $250 \mathrm{MHz}$ parallel RapidIO switch [21]. This test bed was used to calibrate the simulation models and provide the means for more complex simulation experiments using RapidIO hardware and advanced algorithms running on the FPGAs and/or embedded PowerPCs. The simulation work also concerned the RapidIO space systems network fault tolerance. They have presented in $[3,22]$ simulation results of mapping a parallel GMTI application on an embedded multi-processor satellite processing system using a RapidIO interconnection. Three partitioning methods of the real-time GMTI algorithm have been studied. These methods are a straightforward approach, a staggered approach, and a parallel-pipelined approach. These methods were executed on simulated systems of different sizes and topologies. In $[21,23,24]$, they explored considerations for systems capable of executing both GMTI and SAR in real time on an embedded multi-processor satellite system equipped with a RapidIO interconnection network. In [25], they have studied chip level issues associated with the interfacing of a RapidIO network with reconfigurable processing elements through a shared SDRAM interface. They concluded that RapidIO is a promising and viable platform for space-based radar in either GMTI or SAR, with capabilities far exceeding those of traditional busbased systems.

To our best knowledge, Bueno et al. [3,21-25] works are the closest to our research study. They have worked on the modeling and simulation of a multi-processor system based on multiple ASICs processing nodes interconnect by RapidIO bus. The system that we have proposed in this paper consists of two C6678 DSPs, each with eight DSP cores, interconnected with Serial RapidIO bus. Both systems consist of different processing nodes, but both have the same architecture and use RapidIO protocol as an inter-processor communication bus. Also, Bueno et al. [3,21-25] have studied a radar application that has constraints similar to ours in terms of high-processing power and harsh real-time needs. Finally, they have studied a straightforward approach to distribute the radar processing. Simulation results show that this approach provides the best performance on a RapidIO system. It produces the lowest latency, but the parallel efficiency obtained does not exceed $40 \%$ as reported in [3]. By using the straightforward approach, data must be reorganized between processing stages (corner turn). In each corner turn, all processing elements must exchange data between each other which implies all-to-all communications. This limits the parallel efficiency of the system. We have used the same approach to distribute Pulse-Doppler radar signal processing chain algorithm on the proposed parallel machine. The major contributions of this paper are the optimization of the inter-processor communication generated by the straightforward model in the corner turn stages and the improvement of the parallel efficiency of the system.

\section{Background information on Pulse-Doppler radar}

In this section, we provide backgrounds on the PulseDoppler radar. All processing stages of the Pulse-Doppler radar signal processing chain will be described.

\subsection{Introduction}

The radar model studied in this paper is Pulse-Doppler radar. This type of radar transmits a high-frequency impulse signal of high power, followed by a longer break 
during which the echoes can be received, before a new transmitted signal is sent out. The transmission and the reception are alternatively managed by the same antenna using a duplexer [1]. The radar model studied in this paper is running at $\mathrm{S}$ band at about the frequency of 3 $\mathrm{GHz}$ and using beamforming to control the field of view of the radar. Typical targets of these radars are airplanes, ships, helicopters, and even missiles.

The radial speed and the distance or the height of a target can be determined from the position of the antenna and the propagation time.

Pulse-Doppler radar studied in this paper includes the following:

- A network of uniform linear antenna which converts electrical energy into electromagnetic wave when it is used as a transmitter, or collects an electromagnetic wave from a given direction to convert it into electrical energy when it is used as a receiver. A duplexer manages alternating transmitting and receiving modes.

- A signal processing chain is applied to the received echo to extract target information and differentiate the echo coming from a reflection on the ground, clouds, or vegetation.

The signal processing chain of Pulse-Doppler radar consists of two successive processing stages (Figure 1). The first is the coherent processing, which has as goal to maximize the signal-to-noise ratio (SNR) by focusing on the energy. The second is the post-processing which has as goal to detect and recognize targets.

\subsection{Coherent processing}

Coherent processing consists of three steps: beamforming, pulse compression, and Doppler filtering. The goal of coherent processing is to maximize the SNR by focusing on the energy.

\subsubsection{Beamforming}

Conventional antennas need to move mechanically in order to favor a direction. Antennas with a sensor network can select a field of view by applying beamforming to the received signals $[27,28]$. The input signal composed by the signals received by each sensor is filtered to focus energy on a given angular direction. It is then necessary to generate multiple beams, and therefore several filters to describe the number of desired angular directions, and thus, obtain the desired field of view.

The beamforming is done by acting on the amplitude and the phase at each sensor. An operation of 'delay and sum' is commonly used; delaying incoming signals and summing them together after a multiply operation with a defined weight (Figure 2).

Beamforming algorithm can be illustrated as a complex matrix multiplication: $Y=C X$. The $C$ matrix is composed of the beamforming complex coefficients, reflecting phase-shifting, and weighting operations. $X$ is the input matrix composed by the received signal values from $n c$ sensors $(x 1, x 2, \ldots, x \mathrm{nc}) . Y$ is the output matrix that will contain the formed beams $(y 1, y 2, \ldots, y \mathrm{nb})[2]$.

\subsubsection{Pulse compression}

To detect a target, the radar must emit pulses with a large amount of energy. To meet this need, pulses are very short and have high peak power. This type of signal is generated by tube amplifiers which are the only components able to ensure this power constraint in the microwave range. The disadvantages of this solution are the cost and the durability. Solid state transistors overcome those two drawbacks but do not provide a high peak power. The solution of this problem is to extend the emission time of the pulse and to reduce its peak power while maintaining the same amount of energy contained in each pulse. However, this extension of emission time brings up another problem which is resolution [29].

The detection is obtained by cross-correlation between the transmitted pulse and the received wave [30]. If the pulse sent by the radar is sinusoidal, two closely targets can be confused (Figure 3). To overcome this problem, the transmitted pulse is linearly frequency modulated. The signal thus obtained is called 'Chirp' (Figure 4). This improves the range resolution of the radar. Detection of two closely targets is possible (Figure 5).

Concretely, the cross-correlation is performed in the frequency domain. It is done by performing the product of the FFT of the two signals followed by the inverse fast

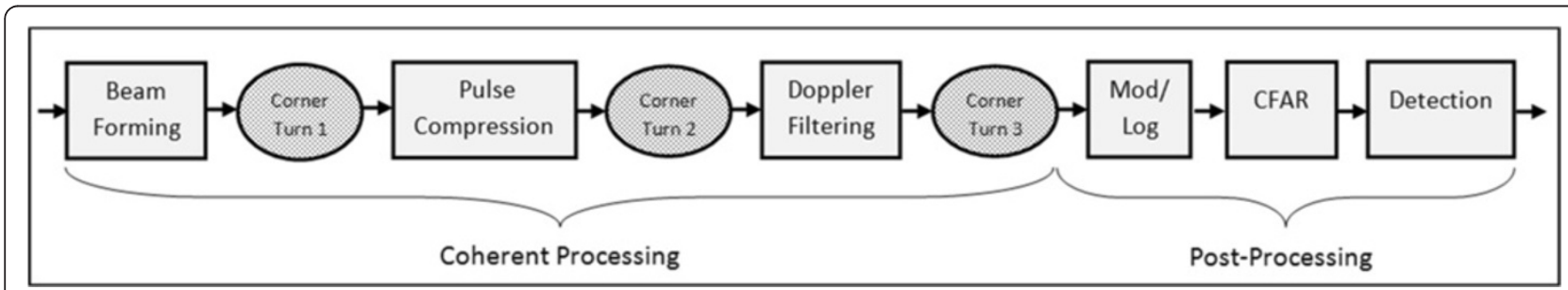

Figure 1 Pulse-Doppler radar processing chain. 


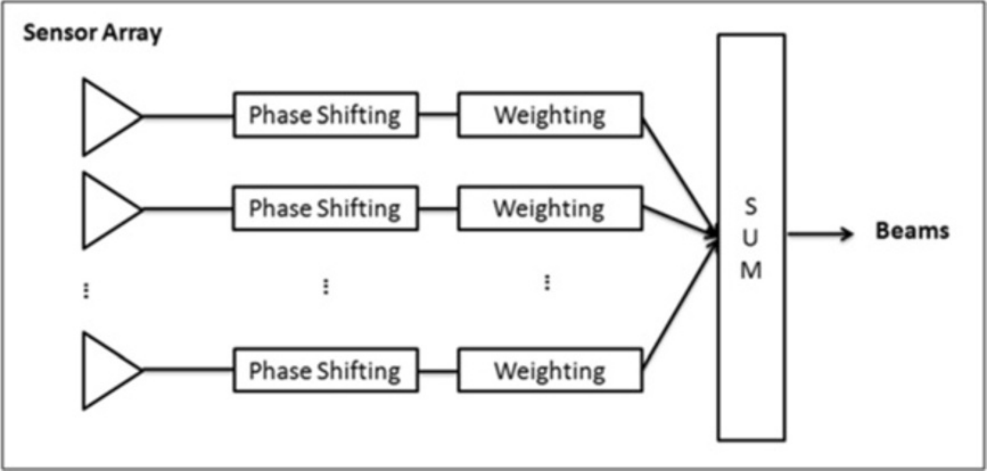

Figure 2 Beamforming structure.

Fourier transform (IFFT) to return to the time domain (Figure 6).

\subsubsection{Doppler filtering}

Based on the Doppler effect, which specifies that the frequency shift of a wave between the transmission and reception is proportional to the speed of the target, this measurement is carried between successive pulses that have a significant phase shift [1]. Each Doppler filter maximizes the SNR on a single Doppler frequency. Detection will then specify this Doppler frequency of the target and measure its radial speed.

Following the same principle as in beamforming, Doppler filtering consists on matrix multiplication: $Y=C X$. The $C$ matrix is composed of the Doppler complex coefficients. $X$ is the input matrix composed by the received pulses values from each sensor $(x 1, x 2, \ldots, x \mathrm{np}) . Y$ is the output matrix that will contains the formed Doppler filters $(y 1$, $y 2, \ldots, y$ nd).

\subsubsection{Corner turn}

The corner turn is an important operation in the signal processing chain of Pulse-Doppler radar. Its goal is to reorganize data between processing tasks in the coherent processing. In order to increase the locality of memory accesses within any given computational stage of the signal processing chain, it is desirable to reorganize the data in memory according to the dimension that the next processing stage will operate along [31].

The corner turn constitutes the only inter-processor communications associated with the algorithm of the signal processing chain of Pulse-Doppler radar. Once all data is organized properly for a given stage of signal processing chain, processing may proceed at each processing element independent of one another in an embarrassingly parallel fashion.

\subsection{Post-processing}

The goal of post-processing is to take decision and detect targets. The post-processing consists of three steps:

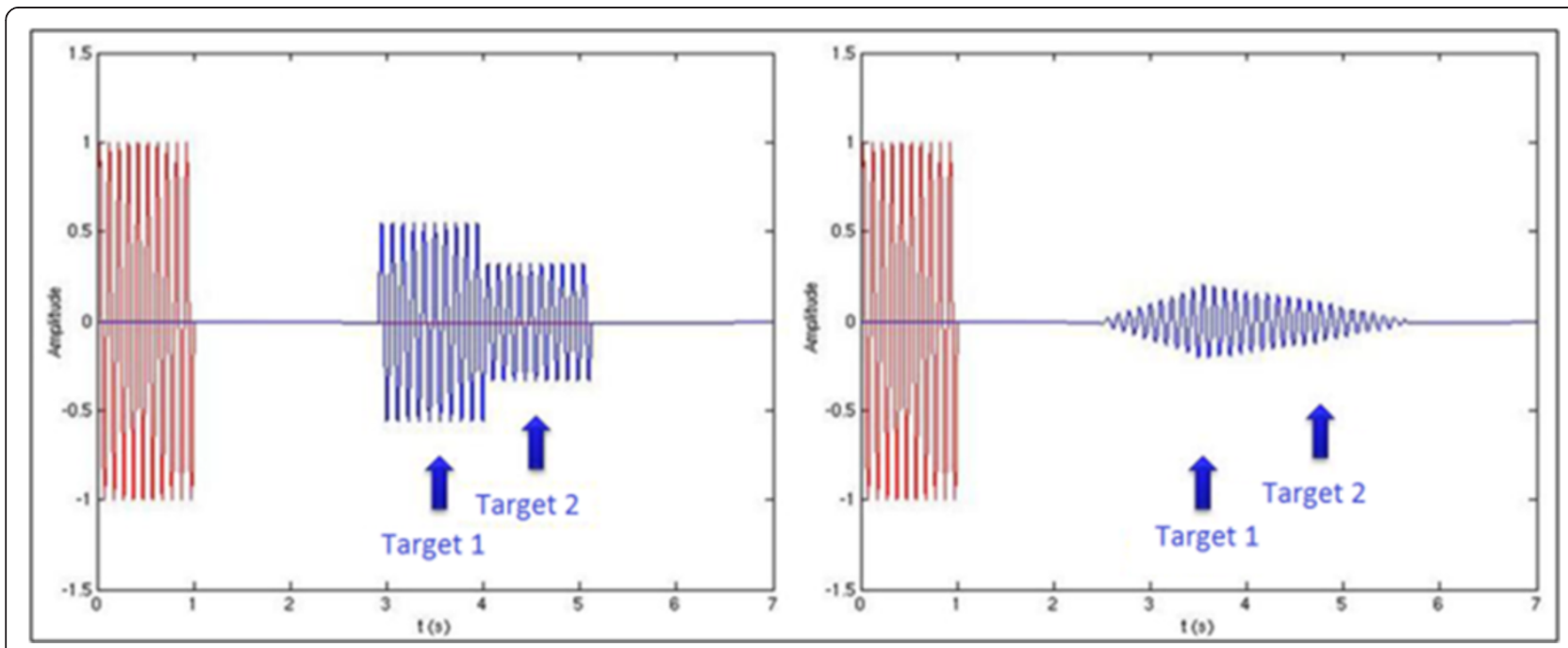

Figure 3 Detection of two closely targets. 


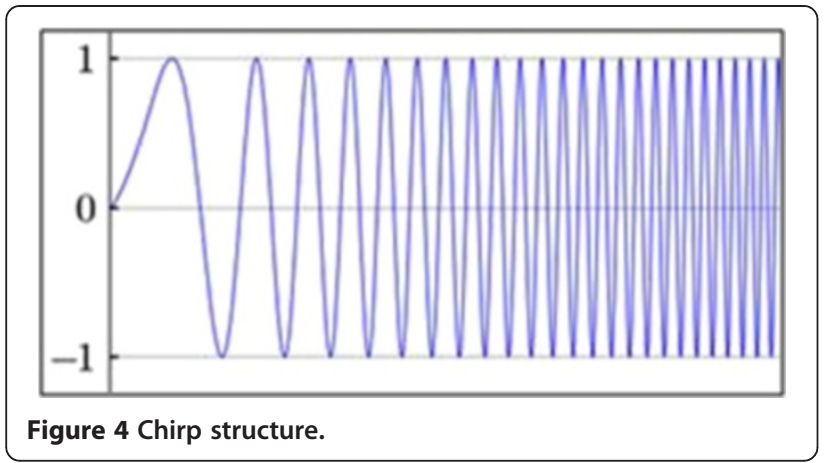

Module/Log, constant false alarm rate (CFAR), and detection. The mapping of the post-processing will not be studied in this paper.

\subsection{Data flow and real-time constraint}

The input data processed by Pulse-Doppler radars is organized as a cube of three dimensions (Figure 7). It is called a burst. The first is the space dimension consisting of all antenna sensors of the radars. The second is the range dimension which represents the time dimension. The third is the Doppler dimension consisting of the number of pulses sent by each sensor.

Parameters of the burst that we have used in our use case are the following:

- Space axis : $\mathrm{Nc}=32$ channels, $\mathrm{Nb}=16$ beams

- Range axis : $\mathrm{Nr}=512$ ranges

- Doppler axis : $\mathrm{Np}=16$ pulses, $\mathrm{Nd}=16$ Doppler filters

Each element in the burst is a complex number coded in 8 bytes as floating number (4 bytes for real part and 4 bytes for imaginary part). The size of the input burst is equal to $2 \mathrm{MB}$.

The bursts arrive from sensors as a stream. The time between the reception of each burst is equal to: $T_{\text {Burst }}=1.64$ ms $\left(T_{\text {Burst }}=\mathrm{Nr} \times \mathrm{Np} \times 200 \mathrm{~ns}\right.$ and the sampling frequency used is equal to $5 \mathrm{MHz}$ (200 ns). The $T_{\text {Burst }}$ is the realtime constraint for the system.

\section{Description of the proposed parallel machine and the experimental platform}

This section describes the experimental platform selected for this research work. We describe the parallel machine architecture; we give an overview about the multi-core C6678 DSP and its evaluation module. Then we describe the RapidIO embedded systems interconnect and the SRIO implemented like a peripheral device in the C6678 DSP. Finally, we provide results of SRIO performances studies performed to define the best way to use this interconnect.

\subsection{Parallel machine's architecture}

The experimental platform proposed in this paper was composed of three multi-core C6678 DSPs connected by SRIO through the SRIO Development Platform Generation 2 (SRDP2) (Figure 8). The processing was distributed on two DSPs (16 DSP cores). One core from the third DSP was dedicated for synchronizing, sending, and receiving bursts.

\subsection{C6678 DSP overview}

The multi-core C6678 DSP provided by Texas Instrument (TI) is a low power and a high-performance fixed/ floating point DSP based on TI's keystone multi-core architecture. It integrates eight C66x DSP cores. Each C66x DSP core can run up to $1.25 \mathrm{GHz}$. The C66x DSP

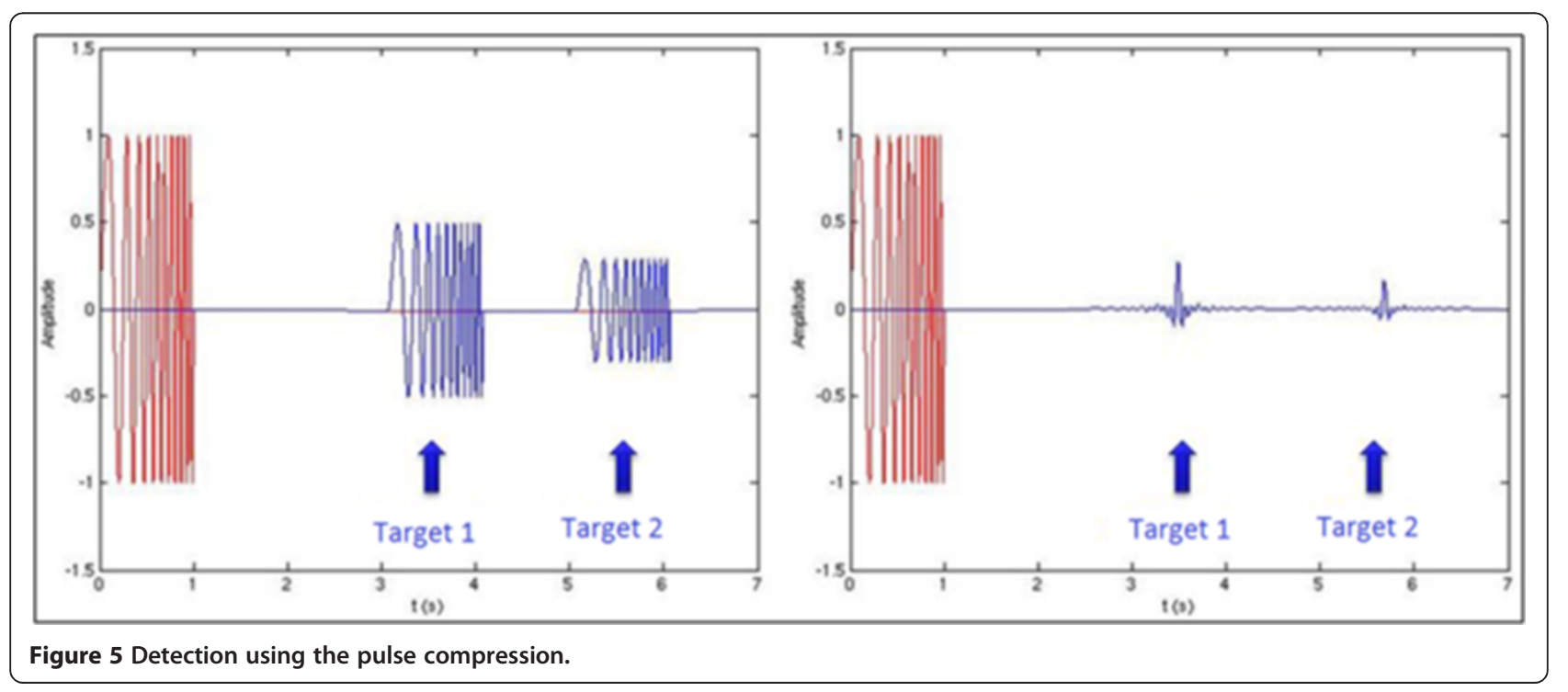




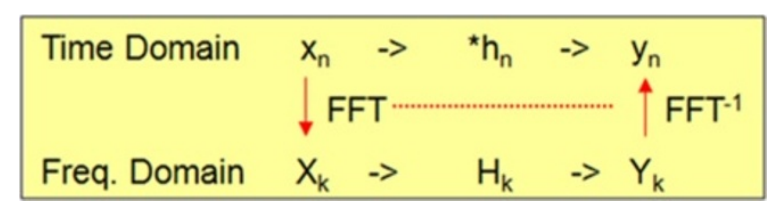

Figure 6 Cross-correlation between time and frequency domain.

core is based on a very long instruction word (VLIW) architecture. The instruction set also includes single input multiple data (SIMD) operating up to 128 -bit vectors. In our test bed, each core run at $1 \mathrm{GHz}$ and dissipates $10 \mathrm{~W}$ of power. With eight cores running at $1 \mathrm{GHz}$, the $\mathrm{C} 6678$ DSP has a peak performance of about 128 single precision GFLOPS (12.8 GFLOPS/watt). Figure 9 shows the functional block diagram of the device [4].

There are three levels of on-chip memory. Each core has a 32-KB of level 1 for program (L1P) and 32-KB of level 1 for data (L1D). The level 1 is the nearest, and it is usually used as cache memory. Additionally, each core has a local level 2 memory; it is slower than level 1, and its size is $512 \mathrm{~KB}$. The level 3 or multi-core shared memory (MSM) is shared and is concurrently accessed by eight cores; its size is $4 \mathrm{MB}$. In addition to that, up to 8-Gbyte external DDR3 RAM can be accessed by the C6678 DSP through a 64-bits bus. The evaluation module EVMC6678 that we have contains 512 Mbytes of DDR3 RAM [32].

The C6678 DSP integrates several high-performance peripherals. It contains three EDMAs to transfer data between memories of the DSP without involving CPU; its bandwidth is about 15 GBPS. It also contains four lanes of the high-performances intercommunication bus SRIO Gen 2 with a baud rate up to 5 Gbps per lane, two

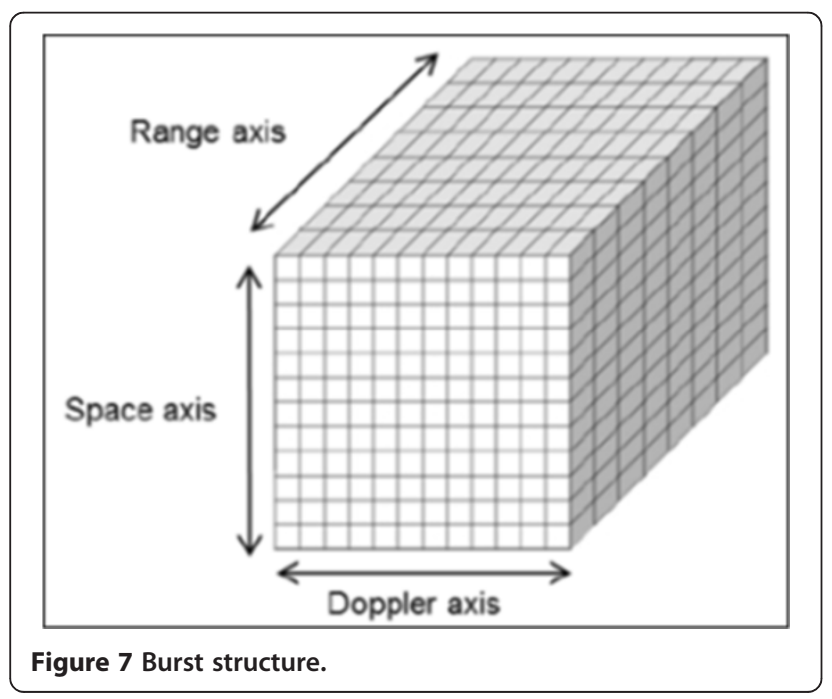

lanes of PCIe Gen2 with a baud rate up to 5 Gbps per lane, and two lanes of gigabit Ethernet.

The EVM6678 evaluation module used in our test bed is a standalone development platform. It has a single-wide equivalent connectors PICMG ${ }^{\circ}$ AMC.0 R2.0 AdvancedMC module [32] (Figure 10). It contains one TI multi-core C6678 DSP, 512 Mbytes of DDR3-1333 memory, 64 Mbytes of NAND flash, and $16 \mathrm{MB}$ SPI NOR flash. The four lanes of SRIO on the C6678 DSP are connected to the AMC port.

\subsection{Description and performance evaluation of SRIO interconnect \\ 4.3.1 RapidIO standard}

The RapidIO interconnect is an open standard controlled by the RapidIO Trade Association. It is a highperformance, packet-switched protocol that was developed to address the embedded industry's need for faster bus speeds, increased bandwidth, reliability, cost effectiveness, and scalability. The RapidIO interconnect allows chip-tochip, board-to-board, and chassis-to-chassis communications at performance levels scaling from 1 gigabit per second per link up to 20 Gbps [13].

The RapidIO standard is defined as a three layer architectural hierarchy (Figure 11): logical, transport, and physical. The physical layer specifies electrical signaling and link level handshaking mechanism. The transport layer defines how packets are routed in the switched fabric. The logical layer defines packet type and function.

\section{Physical specification}

Currently, there are two physical layer specifications recognized by the RapidIO Trade Association: 8/16 LP-LVDS and $1 \times / 4 \times$ LP-Serial. The $8 / 16$ LP-LVDS specification is a point-to-point synchronous clock-sourcing DDR interface. The $1 \times / 4 \times$ LP-Serial specification is a point-to-point, AC coupled, clock-recovery interface. The two physical layer specifications are not compatible.

\section{Transport specification}

The transport layer is used to define how packets are routed in a RapidIO switch fabric. In RapidIO protocol, devices are assigned an 8 or 16 bits device ID. This device ID is integrated in the RapidIO packet as part of a header field. RapidIO switches look at an incoming packet's device ID and determine the correct output port based on internal lookup table.

\section{Logical specification}

The RapidIO logical layer defines the packet types that are supported. Actually, there are three main logical layer specifications: I/O systems, message passing, and global shared memory. 


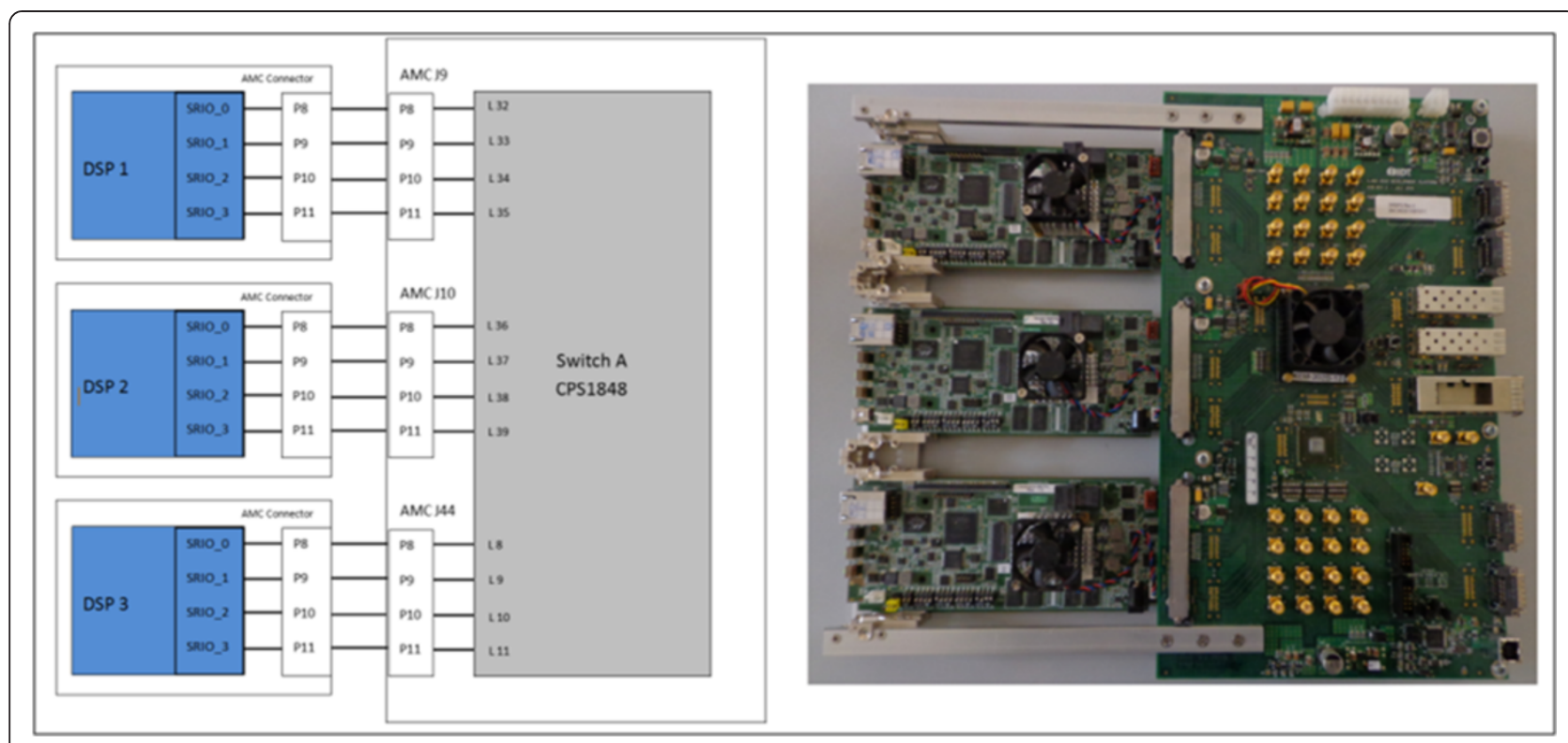

Figure 8 Parallel machine's architecture.

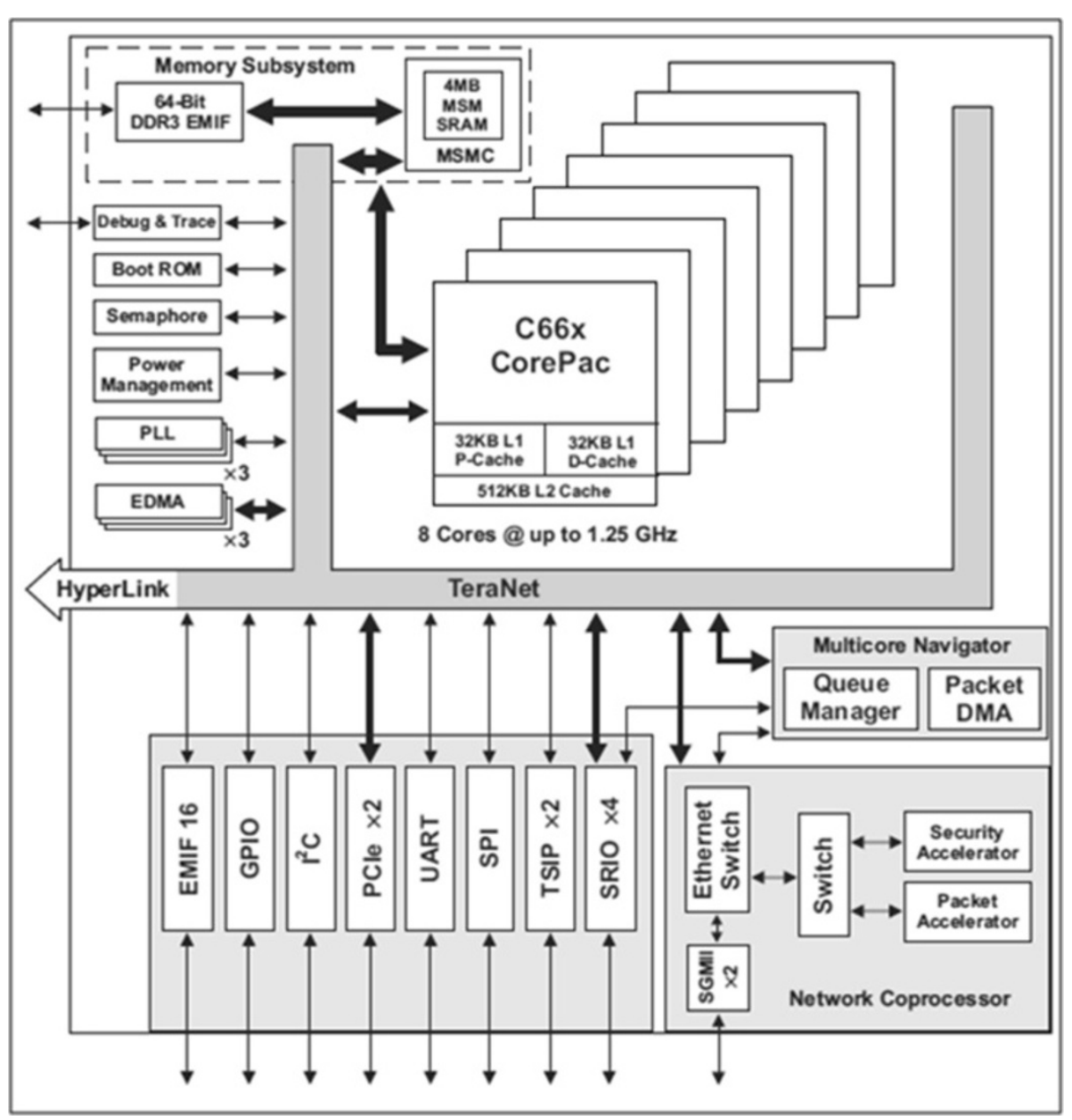

Figure 9 Functional block diagram of the C6678 DSP. 


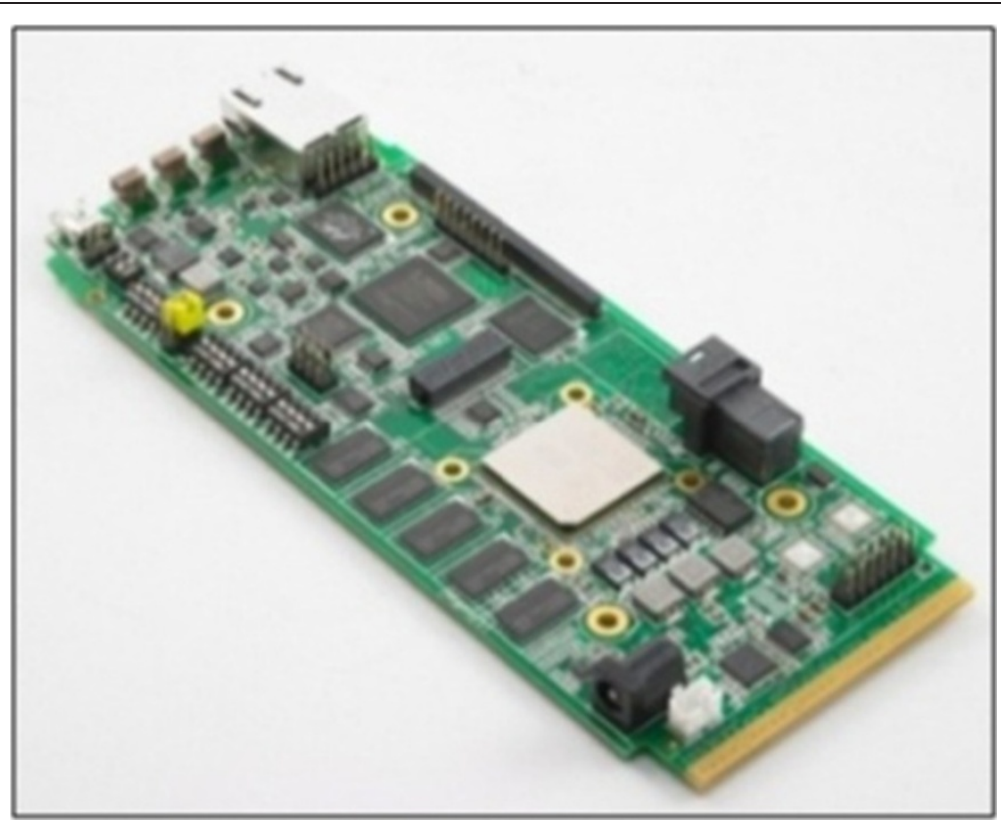

Figure 10 C6678 DSP evaluation module.

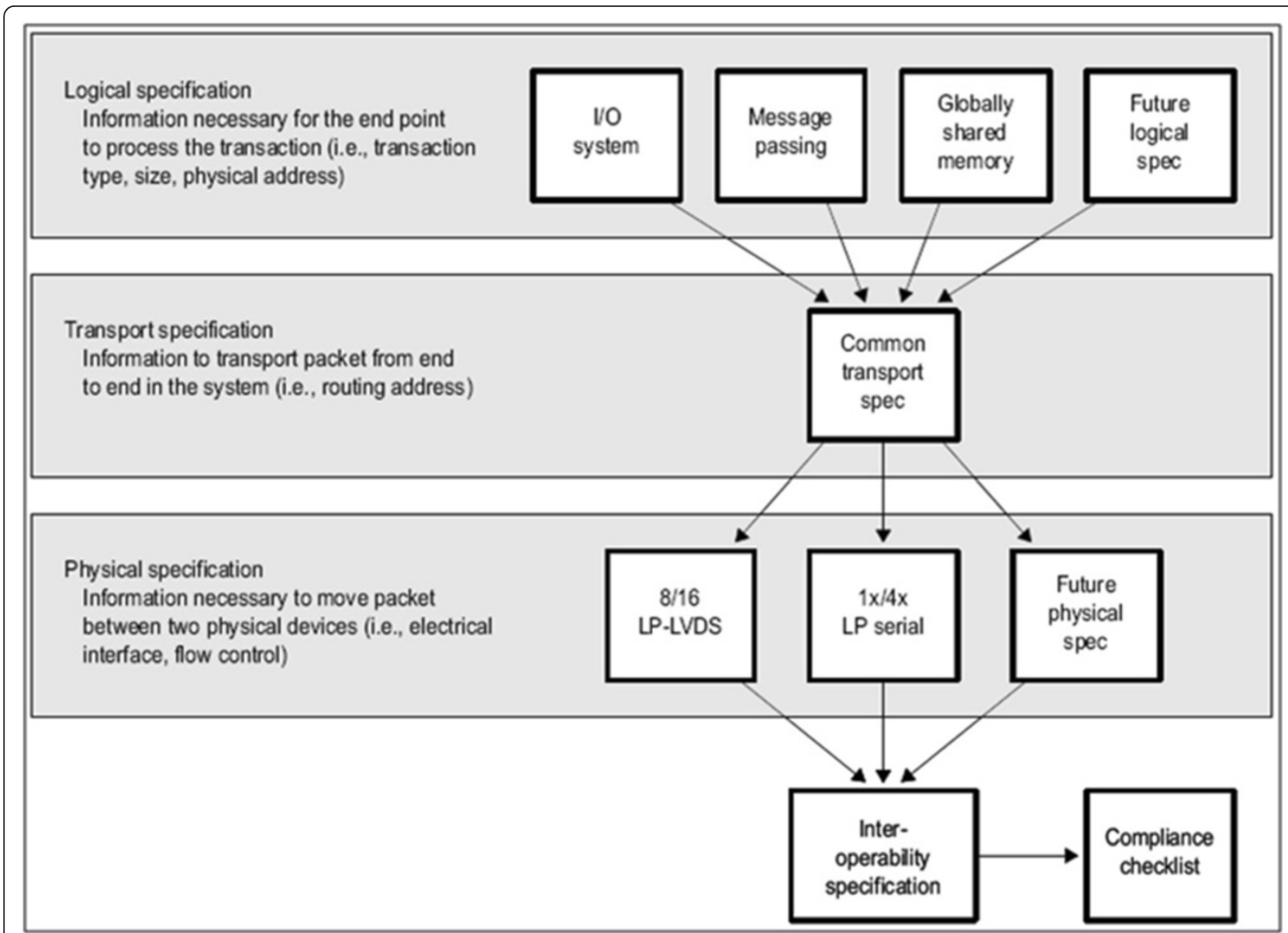

Figure 11 RapidIO architectural hierarchy. 


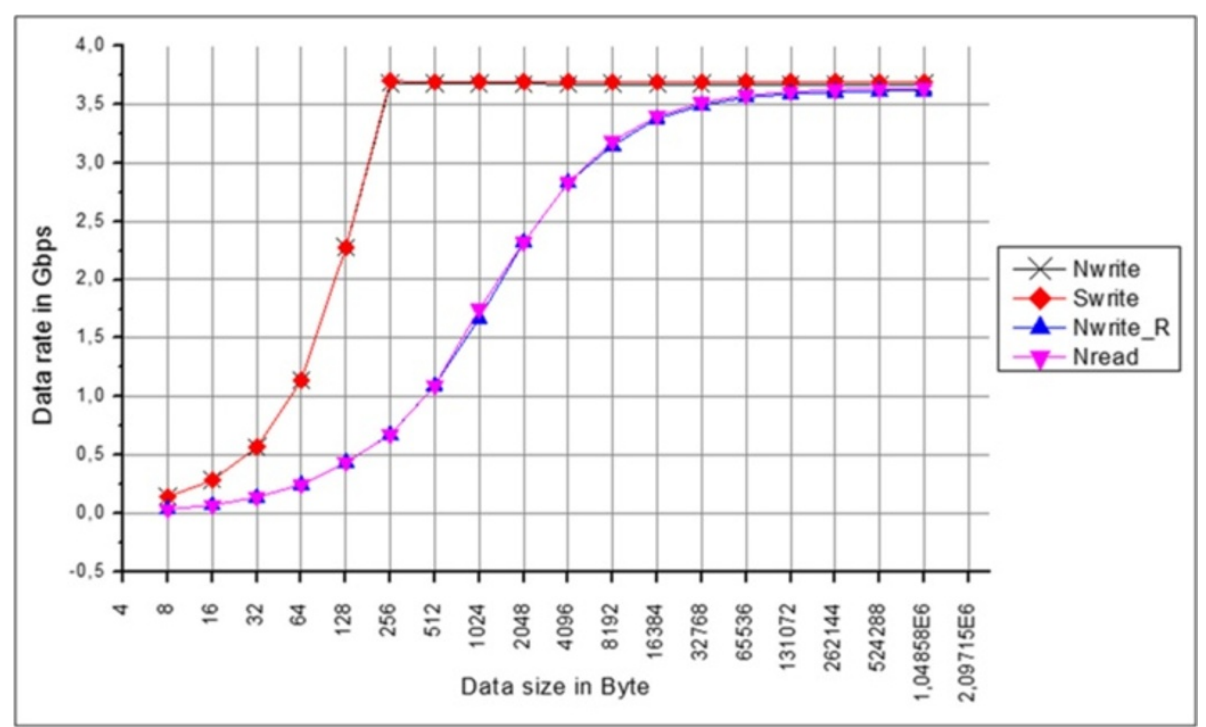

Figure 12 SRIO performances in direct I/O mode with one lane at the baud rate $5 \mathrm{Gbps}$.

The I/O systems specification defines packet types and transactions used for direct memory access (DMA) type transactions. Packet types include operations such as read, write, write-with-response, streaming write, maintenance, and atomic operations. This mode of intercommunication is an extension to a processor's internal DMA. In the I/O systems mode, RapidIO packet contains the specific address where the data should be stored or read in the destination device. Because of that, the I/O systems mode requires that a RapidIO source device keep a local table of addresses for memory within the destination device.

Message passing specification implements a data push model, where data is always written. In this mode, the local device receives a packet and transfers the payload to its memory system.

RapidIO global shared memory logical layer supports cache coherent non-uniform memory access (CC-NUMA) systems [33].

\subsubsection{SRIO implemented in C6678 DSP}

The C6678 DSP includes SRIO like a peripheral device. It implements the RapidIO Interconnect Specification REV2.1.1 compliant. The physical layer supported is the LP-Serial Specification REV2.1.1 compliant [34]; it uses SerDes technology to perform clock recovery from the data stream and incorporates 8-bits/10-bits encoding.

The C6678 DSP integrates four lanes of SRIO. These lanes can operate as one $4 \times$ port, two $2 \times$ port, and four $1 \times$ ports. Each $1 \mathrm{x}$ port can run at frequency of 1.25, 2.5, 3.125, and 5 Gbps. Due to the 8-bits/10-bits encoding overhead, the effective data bandwidth per differential pair is $1.0,2.0,2.5$, and 4 Gbps.
The C6678 DSP implements two specifications of the RapidIO logical layer: I/O systems and message passing; global shared memory is not implemented. The I/O systems mode implemented in the C6678 DSP is called direct $\mathrm{I} / \mathrm{O}$.

Direct I/O mode defines six basic I/O operations: Nwrite, Swrite, Nread, Nwrite_R, Atomic, and maintenance RapidIO transaction. In this paper, we were interested in Nwrite, Swrite, Nread, and Nwrite_R. These four transactions can be separated into two groups: posted transactions and non-posted transactions. Posted transactions are Nwrite and Swrite. They are called posted transactions because they do not have responses or receipt confirmation. The Nwrite and Swrite transactions are write and streaming-write operations. The Swrite transaction is a double-word-only version of the Nwrite transaction that has fewer headers. Non-posted transactions are Nread and Nwrite_R. They are called non-posted transactions because they have responses. These responses may

Table 1 Execution time on a single DSP core

\begin{tabular}{lcc}
\hline Processing stage & $\begin{array}{c}\text { Execution time } \\
\text { measured before } \\
\text { optimization }(\boldsymbol{\mu s})\end{array}$ & $\begin{array}{c}\text { Execution time } \\
\text { measured after } \\
\text { optimization }(\boldsymbol{\mu s})\end{array}$ \\
\hline Beamforming & 4088 & 4261 \\
Corner turn 1 & 5377 & 0 \\
Pulse compression & 5907 & 5907 \\
Corner turn 2 & 1060 & 1060 \\
Doppler Filtering & 2524 & 2697 \\
Corner turn 3 & 5373 & 0 \\
Total & 24329 & 13925 \\
\hline
\end{tabular}




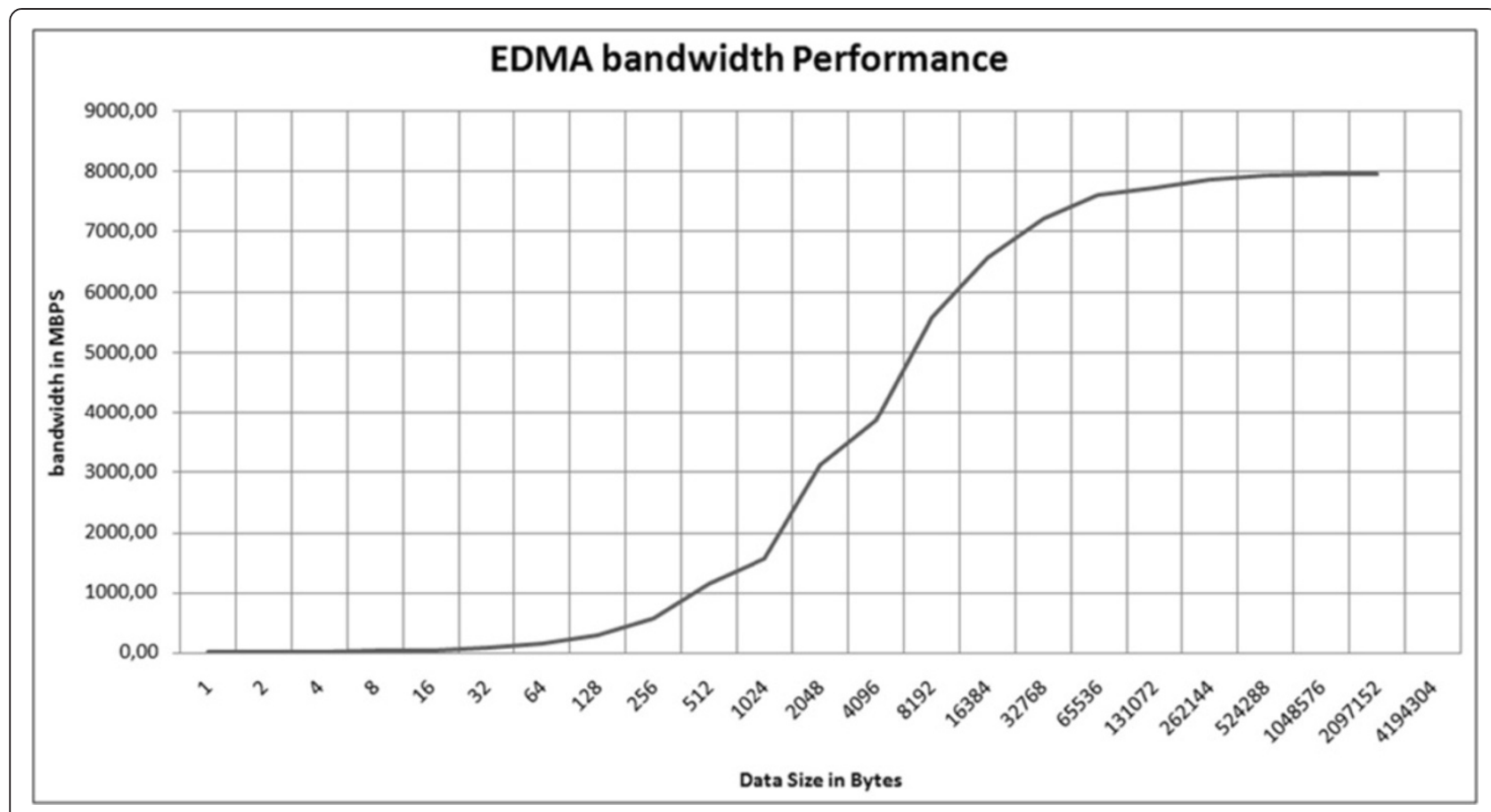

Figure 13 EDMA performance.

contain data as in the case of a reading transaction or not in the case of a write transaction. The Nread transaction is a read operation. The Nwrite_R transaction is a writewith-response operation.

\subsubsection{SRDP2 overview}

The SRDP2 from Integrated Device Technology (IDT) is a flexible test platform for SRIO Gen2 switches. It contains two SRIO switches (CPS1848 and SPS1616), three AMC connectors, two SFP + connectors, one QSFP connector, four InfiniBand/CX4 connectors, and many SMA arrays [35].

Each AMC connector of the SRDP2 contains four lanes of SRIO from the CPS1848 switch. By connecting three evaluation modules EVM6678 to the three AMC port of the SRDP2, the four lanes SRIO of each C6678 DSP will be connected to the CPS1848 switch (Figure 8).

\subsubsection{SRIO performances optimization}

SRIO proposes various communication modes and transaction types in its logical layer. Because of that it was necessary to evaluate the performances of the interconnect and to define the best way to use it.

We have done a series of measurements to evaluate the SRIO performances in direct I/O mode. We have measured performances of posted transactions (Swrite and Nwrite) and non-posted transactions (Nread and Nwrite_R) by sending several packets from one C6678 DSP to another through the CPS1848 SRIO switch using the SRDP2. The transfer was done with different sizes using one SRIO lane at the maximal baud rate supported which is 5 Gbps. In direct I/O mode implemented by TI, it is possible to transfer up to $1 \mathrm{MB}$ of data using a single transaction.

Results presented in Figure 12 show that posted transactions achieve better performances than non-posted transactions when the size of the transferred data is less than $64 \mathrm{~KB}$. This result is due to the fact that non-posted transactions require waiting for a response. But when size of the transferred data is greater than $64 \mathrm{~KB}$, performances of posted and non-posted transactions are very close. This result can be explained by an implementation improvement, performed by TI to optimize the SRIO on the C66x

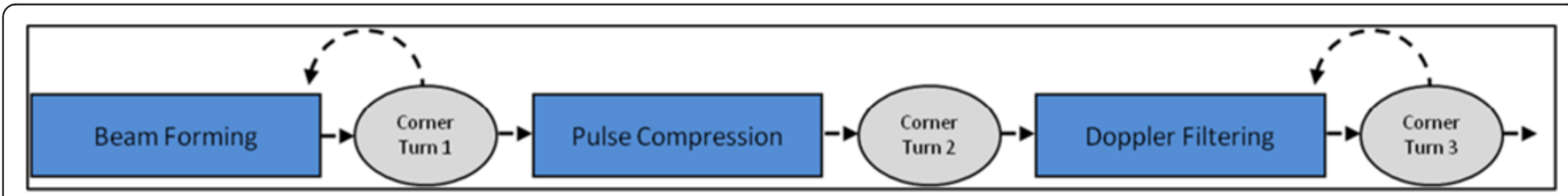

Figure 14 Optimization of the use case implementation on a single DSP core. Optimization of the use case implementation on a single DSP core by integrating corner turn stages 1 and 3 to the processing. 


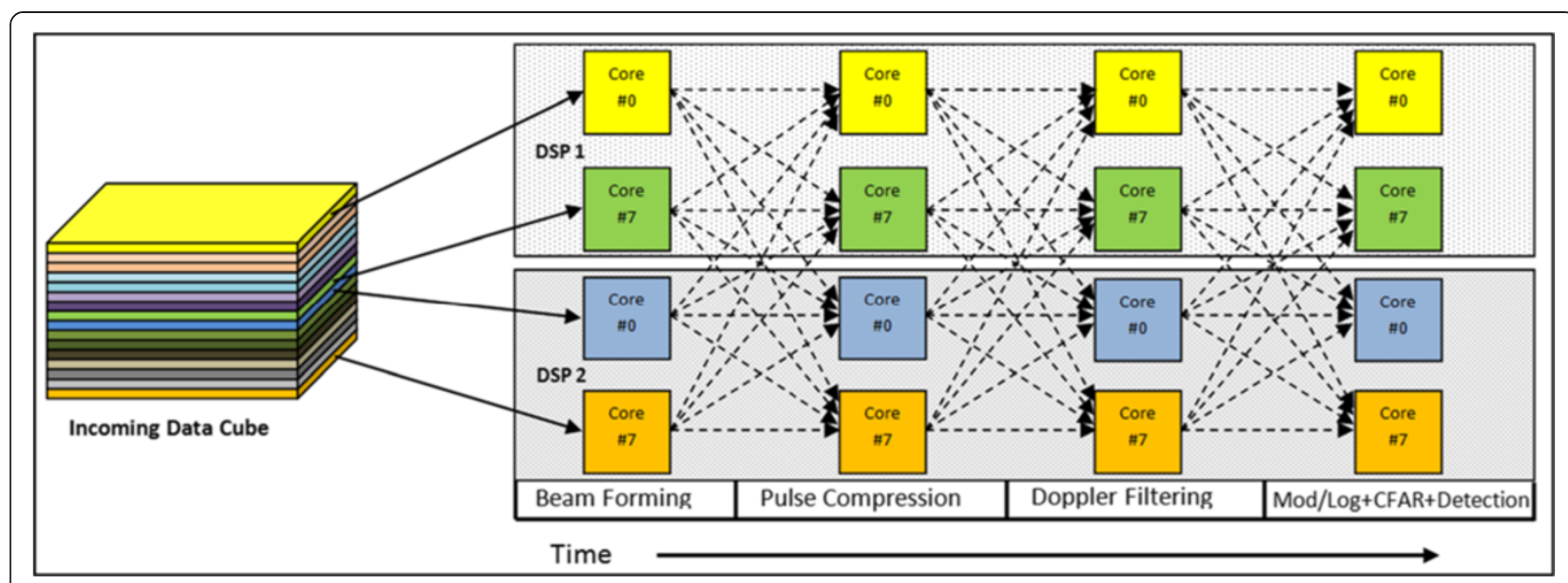

Figure 15 Data flow in the straightforward model.

DSP family, that allow better throughput for non-posted transactions with higher packet size.

The Swrite transaction is a little more efficient than the Nwrite transaction. Its header is smaller than the Nwrite transaction. The Swrite transaction achieves a maximal data rate of 3.70 Gbps while the Nwrite transaction achieves a maximal data rate of $3.67 \mathrm{Gbps}$.

When the size of the transferred data is greater than 256 bytes, the Swrite transaction achieves its maximal data rate. But when the size of the transferred data is lower than 256 bytes, the Swrite transaction performances are degraded. This is due to the fact that the minimal payload that can be sent by one SRIO packet is equal to 256 bytes. So to have greater efficiency with SRIO, the transferred data must be higher than 256 bytes.

By considering the 8-bits/10-bits encoding at the physical layer and the 20 bytes of overhead added to the payload, the theoretical data rate at the baud rate $5 \mathrm{Gbps}$ is equal to $3.71 \mathrm{Gbps}$. The data rate measured with Swrite transaction is very close to the theoretical data rate.

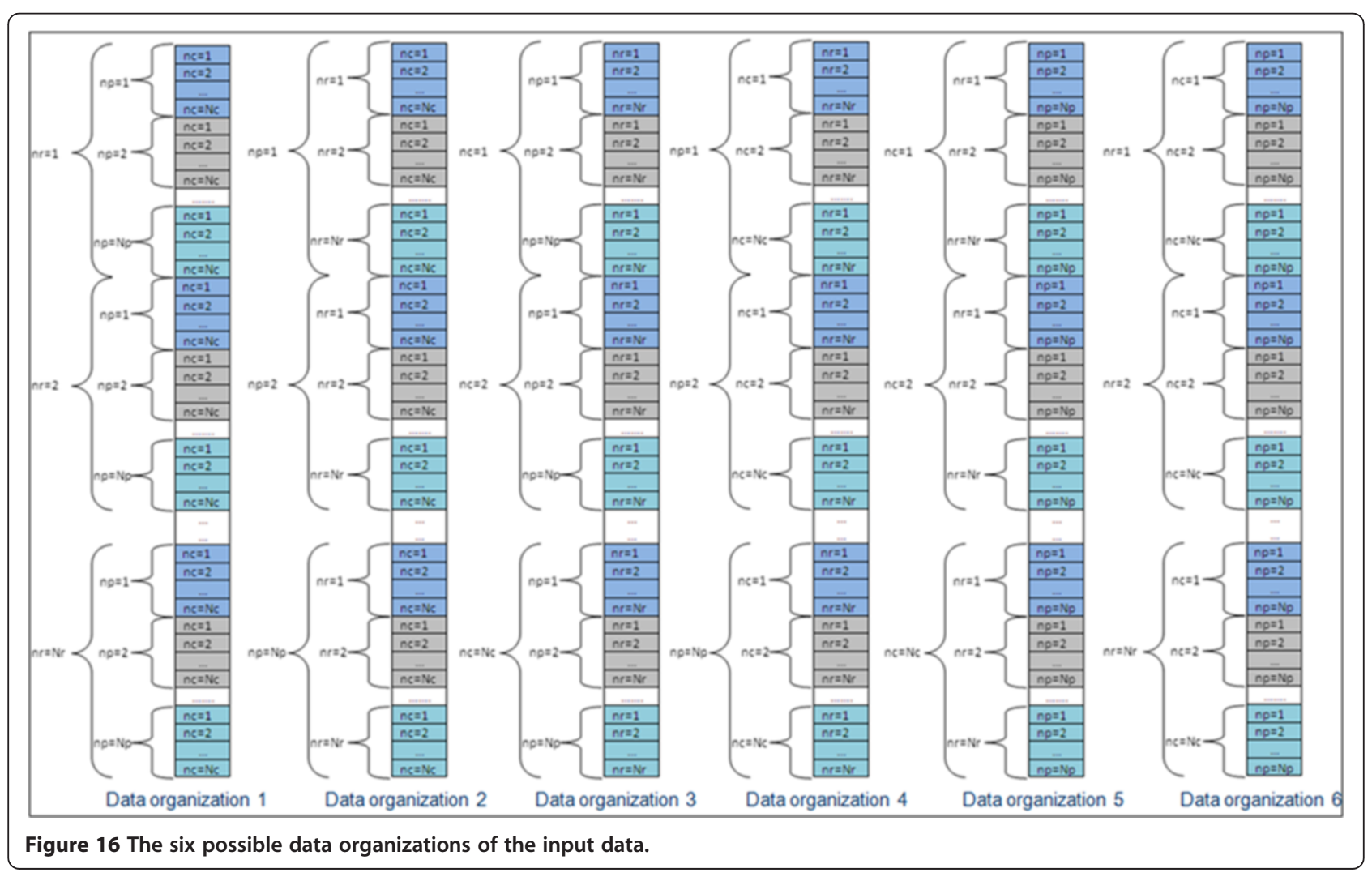


Following this performance evaluation studies, the Swrite transaction has been chosen for data transfers greater than 256 bytes between all DSPs at high data rate.

\section{Optimization of Pulse-Doppler radar processing to parallel architecture}

In this section, we present our experiments and research results that we have performed to map the Pulse-Doppler radar signal processing chain on the proposed parallel machine. We present mapping results on a single DSP core to prove the need of parallel processing. Then, we introduce the straightforward model used to distribute the Pulse-Doppler radar signal processing chain on the parallel machine, and we present all optimizations that we have proposed to reduce the communication time by SRIO. Finally, we give implementation results.

\subsection{Mapping results of the use case on a single DSP core} We start our mapping study by implementing the use case processing on a single core of the C6678 DSP. Code development has been done using the integrated development environment (IDE) Code Composer Studio (CCS) provided by the TI. All optimization levels were active for the TI compiler (7.3.2 version). Due to the size taken by the input burst, all input and output bursts have been placed in MSM memory, while coefficients are in L2 memory. L1 has been fully activated as cache.

Beamforming and Doppler filtering, which are based on a matrix product, have been implemented and optimized using C-intrinsic SIMD instructions. TI C-intrinsic allows access to low-level assembly and constrains the compiler to choose specific hardware features. The pulse compression is based on FFT and IFFT algorithms.

Before optimization, all corner turn stages have been implemented using the EDMA engine. Column 2 in Table 1 shows obtained results. The EDMA controller did not achieve high-performances to do corner turn stages because it must move a large amount of small data blocks. Each small data block consists of 8 bytes: 4 bytes for real part and 4 bytes for imaginary part. We measured the performance of the EDMA when moving different data sizes between two memories (Figure 13), and indeed, there is a performance degradation for a small data size.

We have optimized the use case implementation by integrating the corner turn 1 to beamforming and the corner turn 3 to Doppler filtering (Figure 14). In each processing

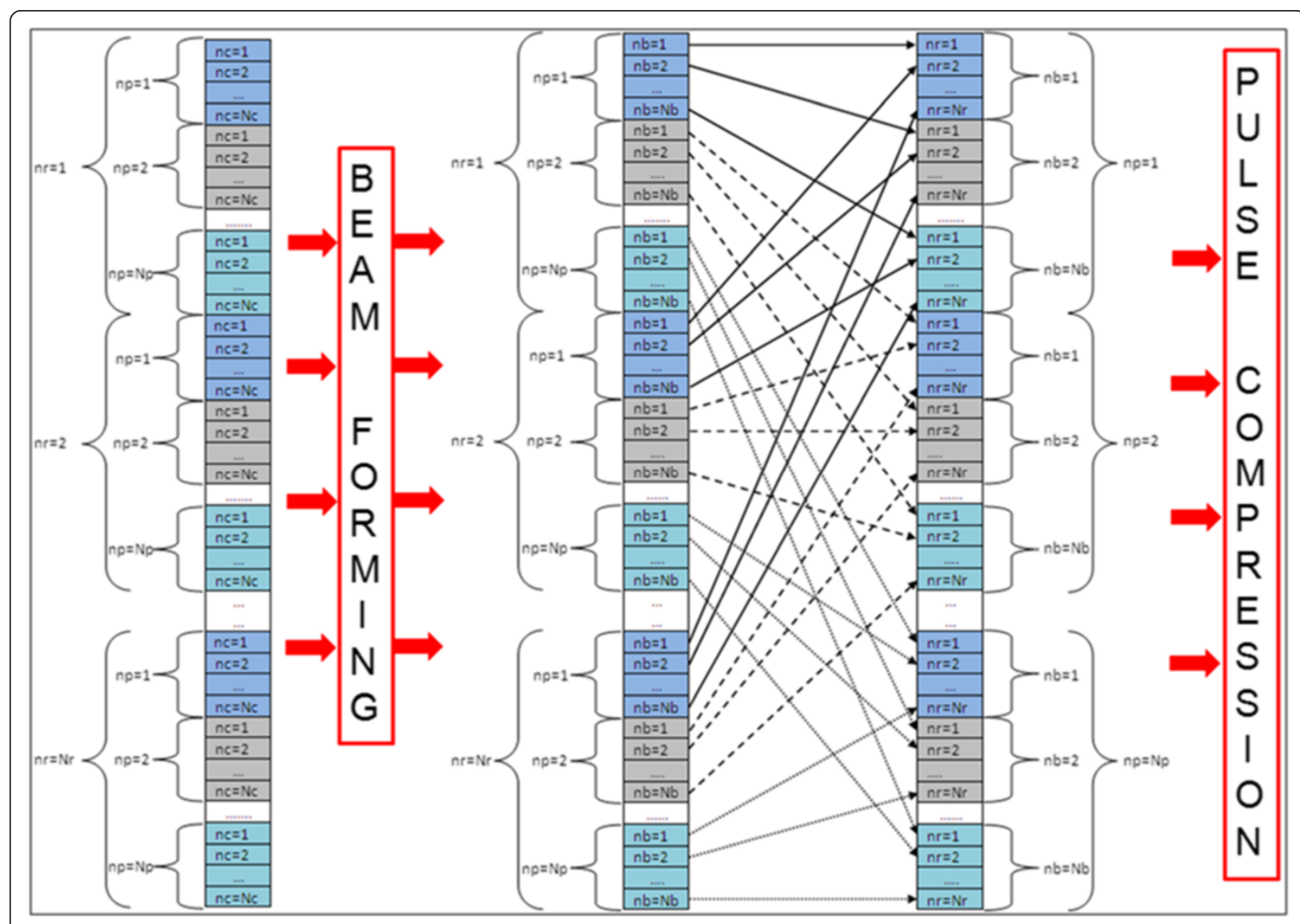

Figure 17 Data flow between the beamforming and pulse compression in the straightforward model before optimization. 
Table 2 Communication time between the beamforming and pulse compression before optimization

\begin{tabular}{lc}
\hline Parameters & Values \\
\hline Number of SRIO packets to exchange between two DSP cores & $\mathrm{Nr} / 16 \times \mathrm{Nb}$ \\
Size of each SRIO packet & 8 bytes \\
Time to send each SRIO packet using one lane (see Section 4.3.4) & $560 \mathrm{~ns}$ \\
Communication time between two DSP cores using one SRIO lane & $\mathrm{Nr} / 16 \times \mathrm{Nb} \times 560 \mathrm{~ns}=287 \mu \mathrm{s}$ \\
Communication time between one DSP core and the 16 other DSP cores using one SRIO lane & $287 \mu \mathrm{ss} \times 16=4.59 \mathrm{~ms}$ \\
Total communication time between the two DSPs using one SRIO lane & $4.59 \mathrm{~ms} \times 8 \mathrm{cores}=36.72 \mathrm{~ms}$ \\
Total communication time between the two DSPs using the four SRIO lane available & $36.72 \mathrm{~ms} / 4=9.18 \mathrm{~ms}$ \\
\hline
\end{tabular}

loop iteration of both beamforming and Doppler filtering, instead of storing the calculated burst elements successively in the output buffer as an image of the input buffer, we have added instructions that calculate and store intended final position of each burst element in the output buffer. Although these added instructions cause an overhead in the processing time, it is still much lower than performing data rearrangement by the EDMA. Corner turn 2 cannot be optimized nor integrated to the pulse compression because the latter is based on FFT and IFFT routines, which compute in each iteration a data block of $\mathrm{Nr}$ burst elements.
The execution time measured after optimization of all signal processing chain is equal to $13.9 \mathrm{~ms}$ (column 3 of Table 1). This value is well above the settled use case real-time constraint of $1.64 \mathrm{~ms}$. Consequently, one single DSP core of the C6678 DSP is not enough to implement the use case processing in real-time fashion.

\subsection{Straightforward mapping model description and optimization}

In order to distribute the Pulse-Doppler radar signal processing chain algorithm on the proposed parallel machine,

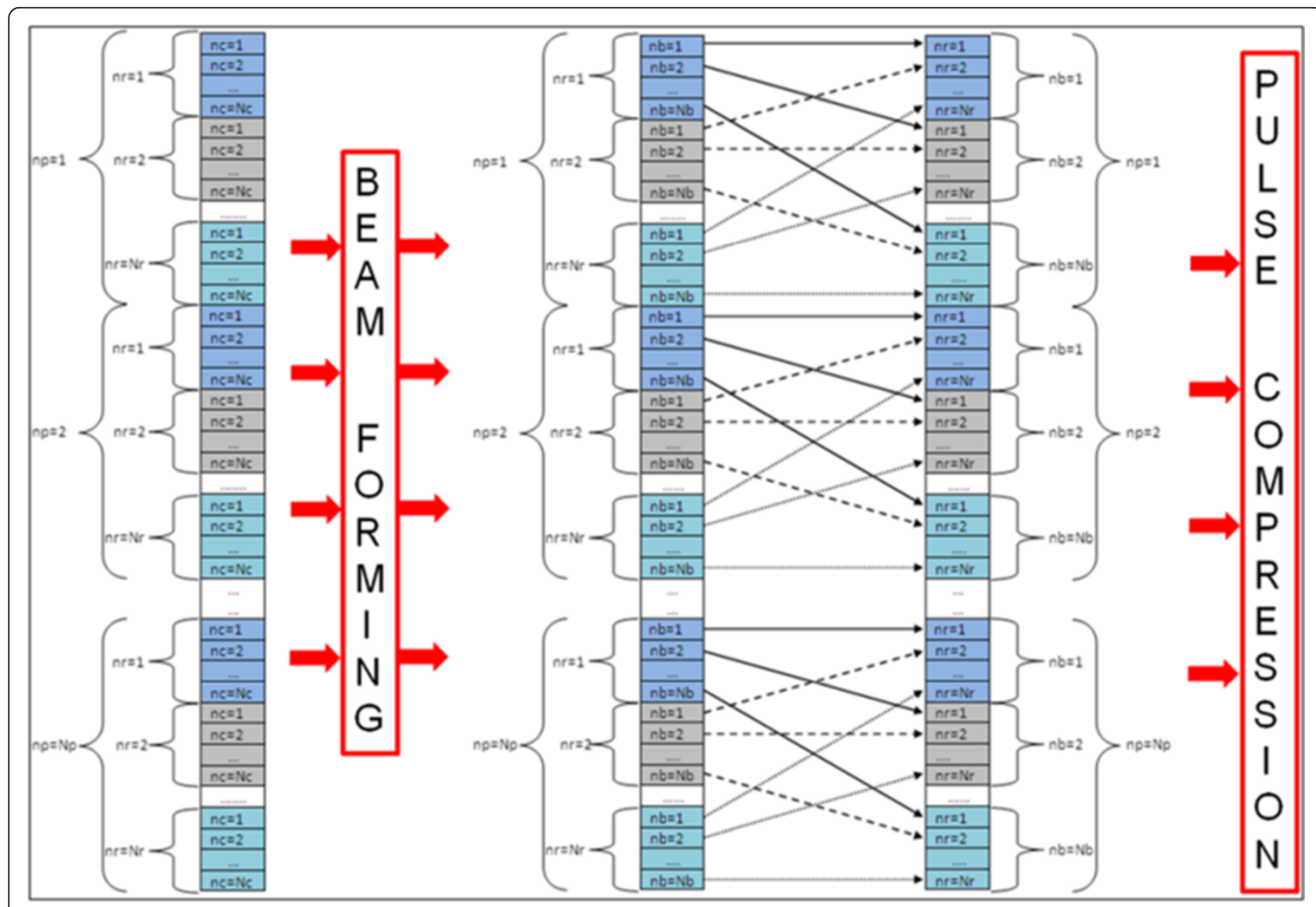

Figure 18 Data flow between the beamforming and pulse compression in the straightforward model after optimization. 
Table 3 Comparison between data rearrangement methods

\begin{tabular}{lccc}
\hline $\begin{array}{l}\text { Data } \\
\text { rearrangement } \\
\text { methods }\end{array}$ & $\begin{array}{c}\text { Data } \\
\text { rearrangement } \\
\text { integrated at the } \\
\text { beamforming }\end{array}$ & $\begin{array}{c}\text { Data } \\
\text { rearrangement } \\
\text { using the EDMA }\end{array}$ & $\begin{array}{c}\text { Data } \\
\text { rearrangement } \\
\text { using CPU }\end{array}$ \\
\hline Measured time $(\mu \mathrm{s})$ & 12 & 300 & 108 \\
\hline
\end{tabular}

we have used a straightforward model (Figure 15) [3,21-25]. In this model, incoming data are distributed equally across each of the DSP cores, and each DSP core performs all stages of the Pulse-Doppler radar processing chain. The straightforward partitioning method provides low latency. The parallel machine must process the entire input data cube before receiving the next cube.

The algorithm of the Pulse-Doppler radar processing chain is composed of functions with no inter-processor communication during each stage. The inter-processor communication is generated in the corner turn stages. Since Pulse-Doppler radar algorithm includes three stages of corner turn, the straightforward mapping model will contain three stages of inter-processor communications. In each stage, each DSP core must exchange data with all other DSP cores which implies an all-to-all communication between DSP cores. This limits the parallel efficiency of the system [3]. We proposed several optimizations to optimize the inter-processor communications by SRIO and to improve the parallel efficiency of the system.

\subsubsection{Optimization of the communications between the beamforming and pulse compression in the straightforward model}

The parallel machine's memory, which is constituted by all processing element memories, has a single dimension. The input data is a three-dimensional cube: Nc indicating the number of the radar antenna sensors, $\mathrm{Nr}$ for ranges axis, and $\mathrm{Np}$ indicating the number of pulses sent by each antenna sensor. To map the input data cube on the parallel machine's memory, input data must be aligned along a single dimension. So, there are six possible data organizations of the input data depending on which dimension input data will be aligned. For example, input data organization 1 is when input data is aligned along Nc dimension as a level $1, \mathrm{~Np}$ dimension in level 2, and $\mathrm{Nr}$ dimension in level 3. Input data organization 2 is as like input data organization 1: input data is aligned along $\mathrm{Nc}$ dimension as a level 1 , but $\mathrm{Nr}$ dimension is in level 2 and $\mathrm{Np}$ dimension in level 3 (Figure 16).

In order to efficiently execute the first stage of the beamforming in the Pulse-Doppler radar signal processing chain, the input data cube must be aligned along Nc dimension to increase the locality of memory accesses and avoid making jumps when reading the data from the memory. Indeed, writing or reading data with jumps from a DDR memory significantly reduces the bandwidth of the DDR memory. Data organizations 3, 4, 5, and 6 are eliminated.

By choosing the data organization 1 of the data cube as input to the beamforming, the data flow between the beamforming and pulse compression is presented in Figure 17.

Partitioning of the beamforming is done along a range dimension. Each DSP core will process $\mathrm{Nr} / 16$ ranges. The SRIO communication time calculated between the beamforming and pulse compression is about $9.18 \mathrm{~ms}$ (Table 2).

After choosing the data organization 2 of the input data cube, the data flow between the beamforming and pulse compression will become as presented in Figure 18. In this case, partitioning of the beamforming is done along pulse dimension. Each DSP core will process one pulse. After the beamforming, DSP cores will not have to communicate

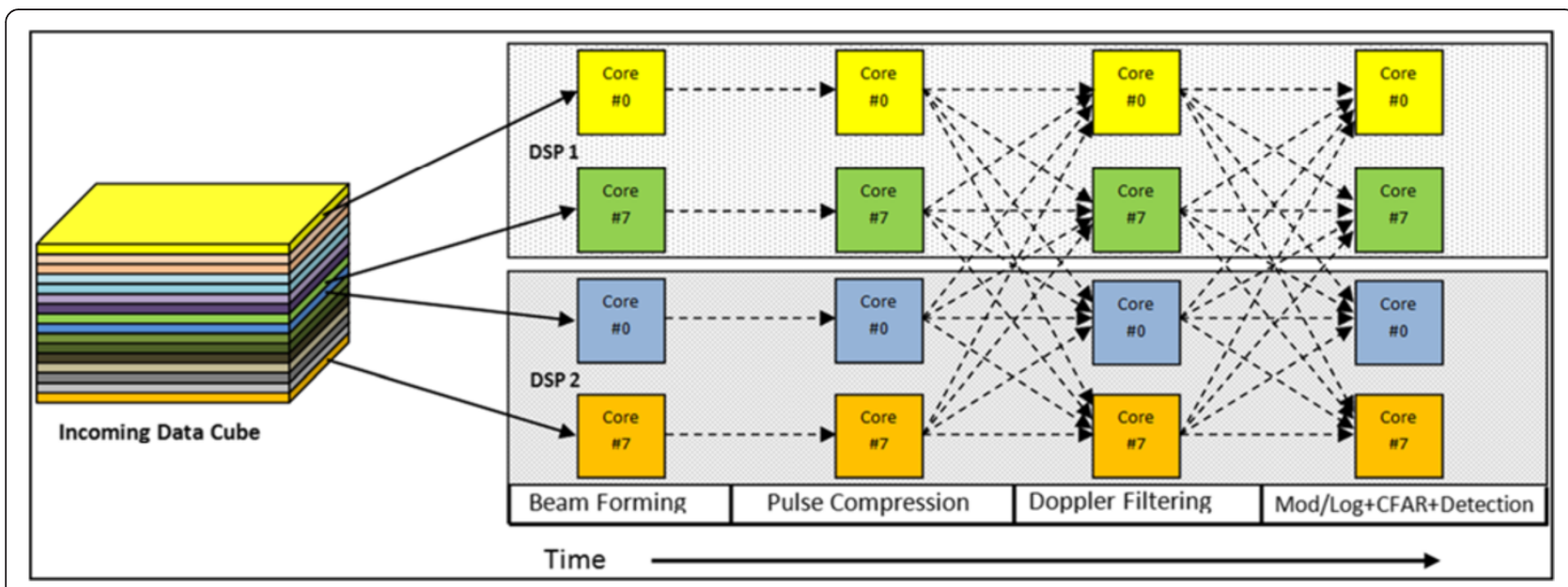

Figure 19 Straightforward model after optimization of the communications between the beamforming and pulse compression. 


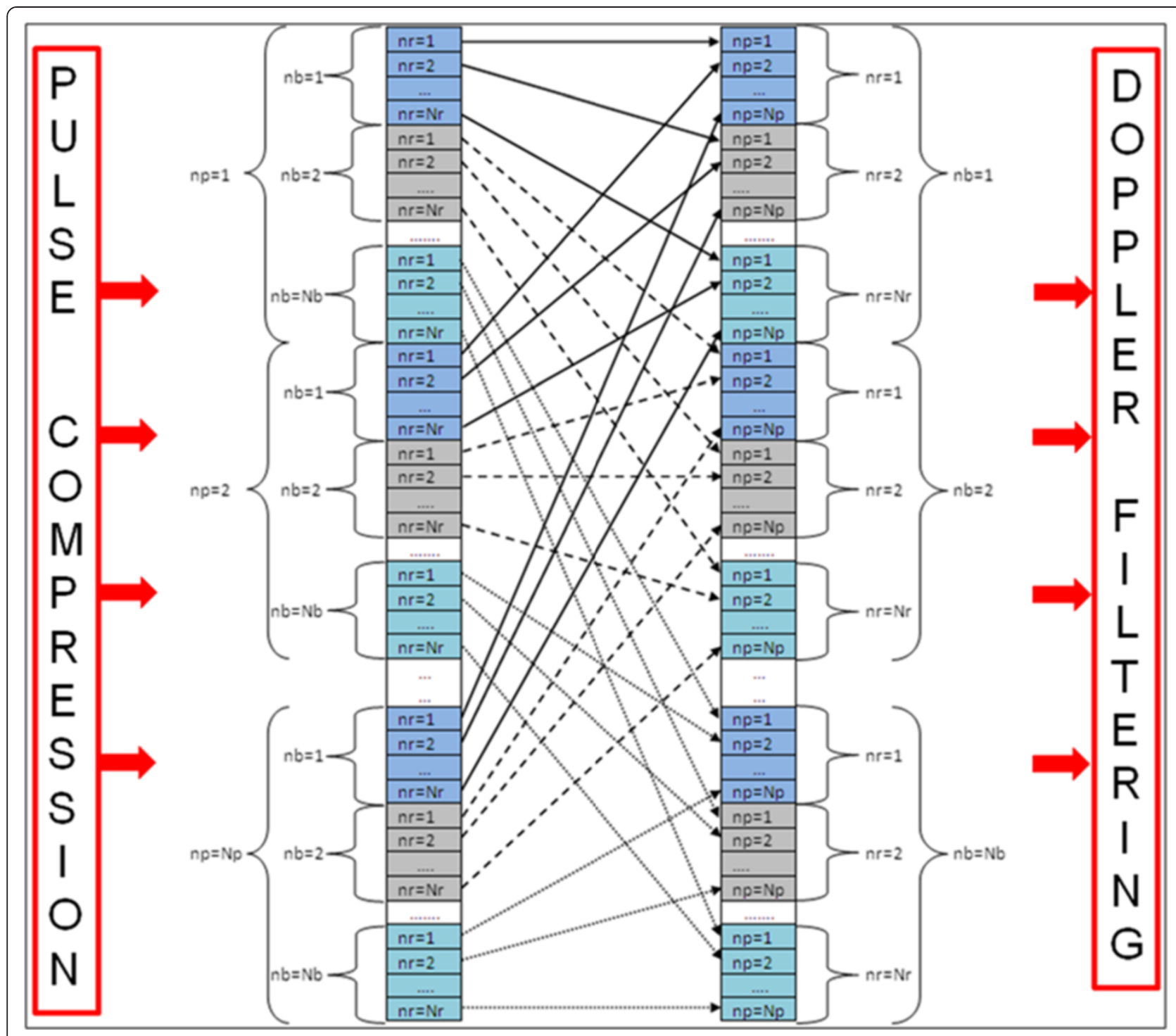

Figure 20 Data flow between the pulse compression and Doppler filtering in the straightforward model before optimization.

Table 4 Communication time between the pulse compression and Doppler filtering before optimization

Parameters

Values

$\mathrm{Nr}$

Number of SRIO packets to exchange between two DSP cores

Size of each SRIO packet

Time to send each SRIO packet using one lane (see Section 4.3.4)

Communication time between two DSP cores using one SRIO lane

Communication time between one DSP core and the 16 other DSP cores using one SRIO lane

Total communication time between the two DSPs using one SRIO lane

Total communication time between the two DSPs using the four SRIO lane available

\section{8 bytes}

$560 \mathrm{~ns}$

$\mathrm{Nr} \times 560 \mathrm{~ns}=287 \mu \mathrm{s}$

$287 \mu \mathrm{s} \times 16=4.59 \mathrm{~ms}$

$4.59 \mathrm{~ms} \times 8$ cores $=36.72 \mathrm{~ms}$

$36.72 \mathrm{~ms} / 4=9.18 \mathrm{~ms}$ 


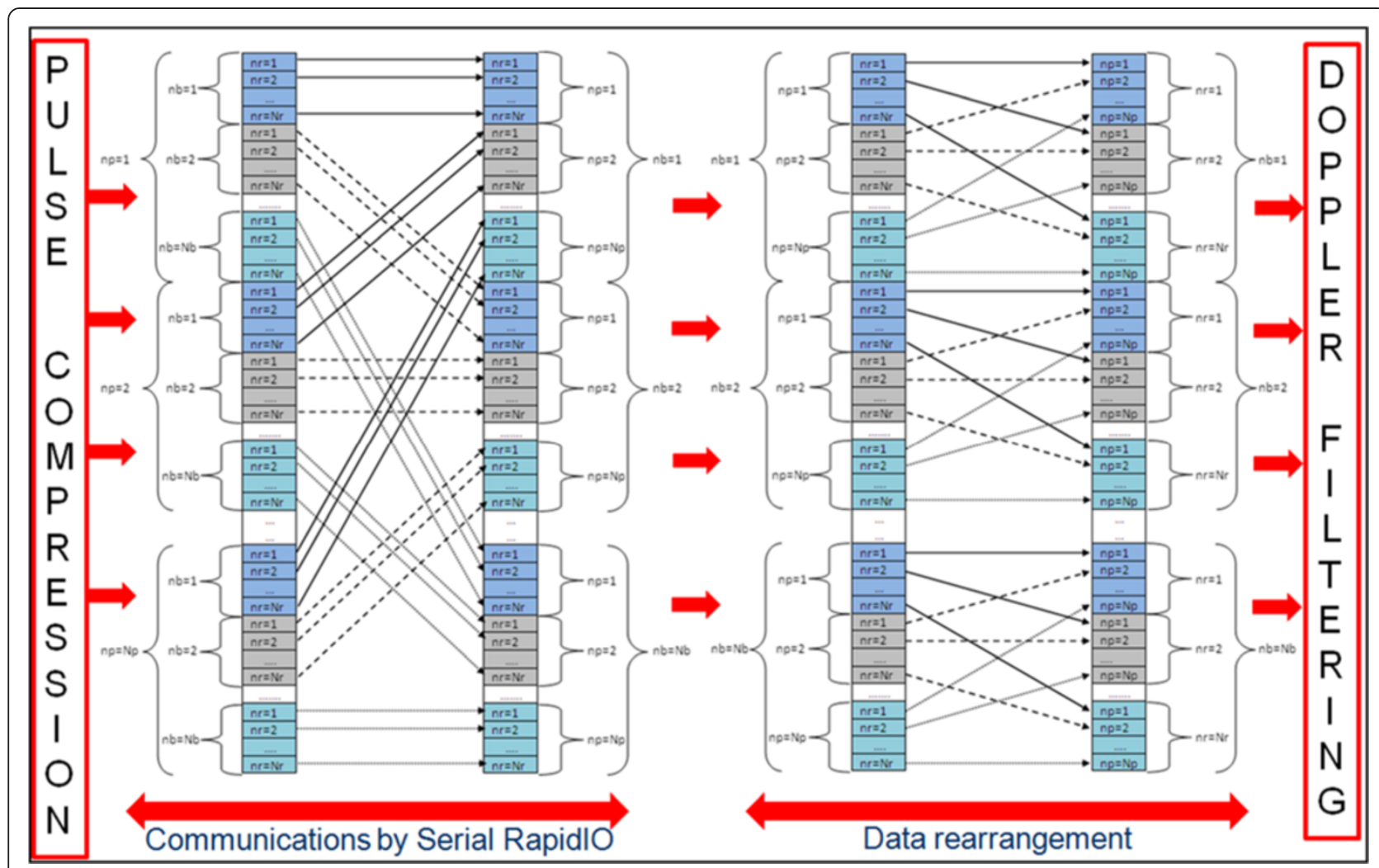

Figure 21 Data flow between the pulse compression and Doppler filtering in the straightforward model after optimization.

between each other. They have just to rearrange data locally before starting the pulse compression. Three methods have been proposed and compared to perform this data rearrangement. The first method is to integrate the data rearrangement at the end of beamforming, the second method is to perform this data rearrangement using the EDMA, and the third one is to achieve it using CPU. From comparison study presented in Table 3, the most efficient way to perform this data rearrangement is to integrate it at the end of beamforming.

As a conclusion, by using the data organization 2 at the input of the beamforming, communications by SRIO between the beamforming and pulse compression has been eliminated (Figure 19).

\subsubsection{Optimization of the communications between the pulse compression and Doppler filtering in the straightforward model}

After choosing the data organization of the input data cube that optimizes communications between the beamforming and pulse compression in the straightforward model, the data flow between the pulse compression and Doppler filtering is presented in Figure 20.

Distribution of the pulse compression is done along the pulse dimension. Each DSP core will process one pulse. The SRIO communication time calculated between the pulse compression and Doppler filtering is about $9.18 \mathrm{~ms}$ (Table 4). Data size sent by each SRIO packet is less than 256 bytes; SRIO is not used in an efficient way. To

Table 5 Communication time between the pulse compression and Doppler filtering after optimization

\begin{tabular}{lc}
\hline Parameters & Values \\
\hline Number of SRIO packets to exchange between two DSP cores & 1 \\
Size of each SRIO packet & $\mathrm{Nr} \times 8$ bytes =4 KB \\
Time to send each SRIO packet using one lane (see Section 4.3.4) & $9 \mu \mathrm{s}$ \\
Communication time between two DSP cores using one SRIO lane & $9 \mu \mathrm{s}$ \\
Communication time between one DSP core and the 16 other DSP cores using one SRIO lane & $9 \mu \mathrm{s} \times 16=144 \mu \mathrm{s}$ \\
Total communication time between the two DSPs using one SRIO lane & $144 \mu \mathrm{s} \times 8 \mathrm{cores}=1.15 \mathrm{~ms}$ \\
Total communication time between the two DSPS using the four SRIO lane available & $1.15 \mathrm{~ms} / 4=288 \mu \mathrm{Ss}$ \\
\hline
\end{tabular}




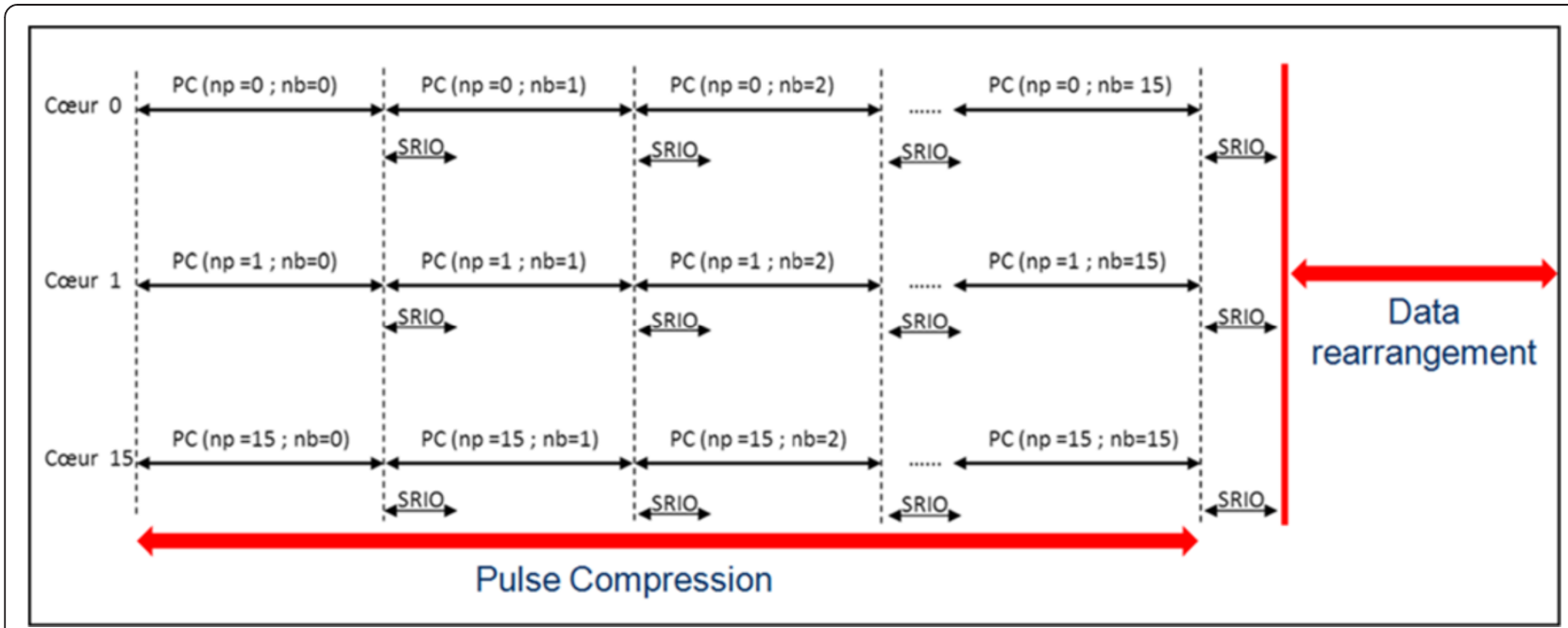

Figure 22 SRIO communications performed in parallel with processing.

overcome this limitation and reduce the communication time by SRIO, we have proposed to add an intermediate stage between the pulse compression and Doppler filtering (Figure 21). The proposed method separates communications by SRIO between the pulse compression and Doppler filtering on two stages. The first stage corresponds to SRIO communications, and the second corresponds to a local data rearrangement.

\section{Communications by SRIO}

In this case, the SRIO communication time calculated between the pulse compression and Doppler filtering is equal to $288 \mu$ s (Table 5). SRIO in the proposed method is used in an efficient way. The data size sent by each packet is more than 256 bytes.

SRIO communications between the pulse compression and Doppler filtering can be overlapped with computation. It can be started in parallel with the pulse compression (Figure 22). Each DSP core in the straightforward model executes the pulse compression of one pulse and $\mathrm{Nb}$ beams. The result of the pulse compression of each beam can be sent by SRIO to another DSP core without having to wait the end of the pulse compression. Using this approach, parallel machine will execute $94 \%$ of communications by SRIO in parallel with the pulse compression.

\section{Data rearrangement}

The second stage in the proposed method corresponds to a local data rearrangement on each DSP core. We have proposed and compared three different methods to perform this data rearrangement. The first method is to integrate this rearrangement at the Doppler filtering, the second method is to achieve it using the EDMA, and the third one is to achieve it using the CPU.
By executing Doppler filtering of one beam on a single DSP core, the executing time when input data is wellarranged is equal to $157 \mu \mathrm{s}$. While when input data is not well-arranged and the data rearrangement is integrated to the processing, the execution time obtained is equal to $514 \mu \mathrm{s}$. This large difference is due to the fact of cache-coherence mechanisms which do not operate well when input data is not well-arranged.

As seen before, the C6678 DSP has three EDMA controllers that can operate in parallel. One EDMA can achieve the data rearrangement of one beam in $73 \mu$ s. Eight beams must be rearranged by each C6678 DSP. The three EDMA available achieve the data rearrangement of eight beams in $300 \mu \mathrm{s}$.

Each DSP core executes data rearrangement of one beam in $108 \mu \mathrm{s}$. Since the eight DSP cores can operate in parallel, the data rearrangement of eight beams can be executed in $108 \mu$ s.

From this comparison study summarized in Table 6, the efficient way to perform the data rearrangement of the second stage in the proposed method is to execute it using CPU.

As a conclusion, the proposed method which separate communications by SRIO between the pulse compression and Doppler filtering into two stages has greatly optimized communication time by SRIO.

Table 6 Comparison between data rearrangement methods

\begin{tabular}{lccc}
\hline $\begin{array}{l}\text { Data } \\
\text { rearrangement } \\
\text { methods }\end{array}$ & $\begin{array}{c}\text { Data } \\
\text { rearrangement } \\
\text { integrated at } \\
\text { the Doppler } \\
\text { filtering }\end{array}$ & $\begin{array}{c}\text { Data } \\
\text { rearrangement } \\
\text { using the EDMA }\end{array}$ & $\begin{array}{c}\text { Data } \\
\text { rearrangement } \\
\text { using CPU }\end{array}$ \\
\hline Measured time $(\mu \mathrm{s})$ & 357 & 300 & 108 \\
\hline
\end{tabular}




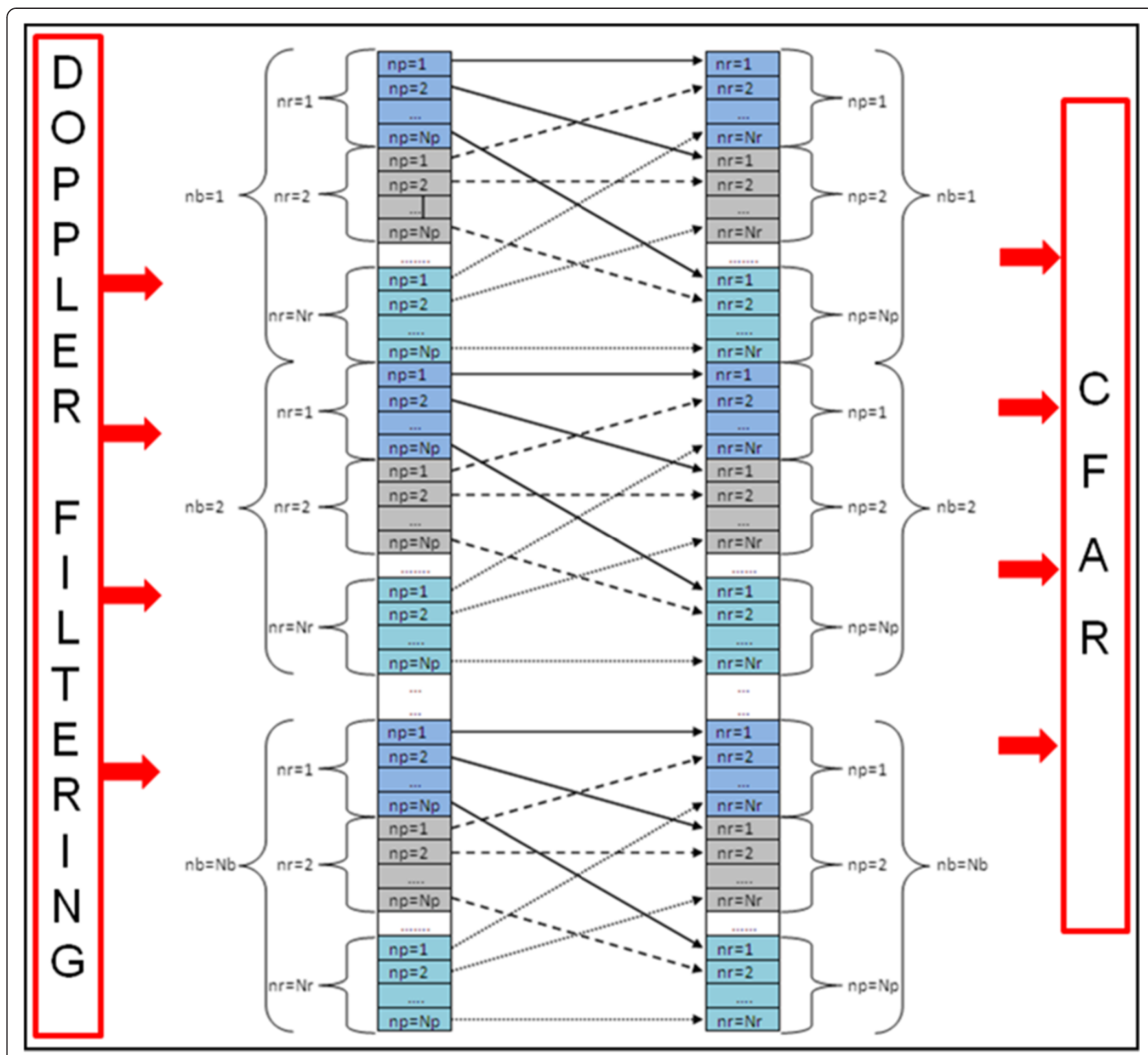

Figure 23 Data flow between the Doppler filtering and the post-processing in the straightforward model.

\subsubsection{Optimization of the communications between the} Doppler filtering and the post-processing in the straightforward model

The data flow between the Doppler filtering and the postprocessing in the straightforward model after choosing a data organization that optimizes the post-processing is presented in Figure 23.

Distribution of the Doppler filtering is done along beam dimension. Each DSP core will process one beam. After Doppler filtering, DSP cores will not have to communicate between each other. They have just to rearrange data locally before starting post-processing. Three methods have been proposed and compared to perform this data rearrangement. The first method is to integrate the data rearrangement at the end of the Doppler filtering, the second method is to perform this data rearrangement using the EDMA, and the third one is to achieve it using CPU. From the comparison study presented in Table 7, the most efficient way to perform this data rearrangement is to integrate it at the end of the Doppler filtering.

Table 7 Comparison between data rearrangement methods

\begin{tabular}{lccc}
\hline $\begin{array}{l}\text { Data } \\
\text { rearrangement } \\
\text { methods }\end{array}$ & $\begin{array}{c}\text { Data } \\
\text { rearrangement } \\
\text { integrated at } \\
\text { the Doppler } \\
\text { filtering }\end{array}$ & $\begin{array}{c}\text { Data } \\
\text { rearrangement } \\
\text { using the EDMA }\end{array}$ & $\begin{array}{c}\text { Data } \\
\text { rearrangement } \\
\text { using CPU }\end{array}$ \\
\hline Measured time $(\mu \mathrm{s})$ & 12 & 300 & 108 \\
\hline
\end{tabular}




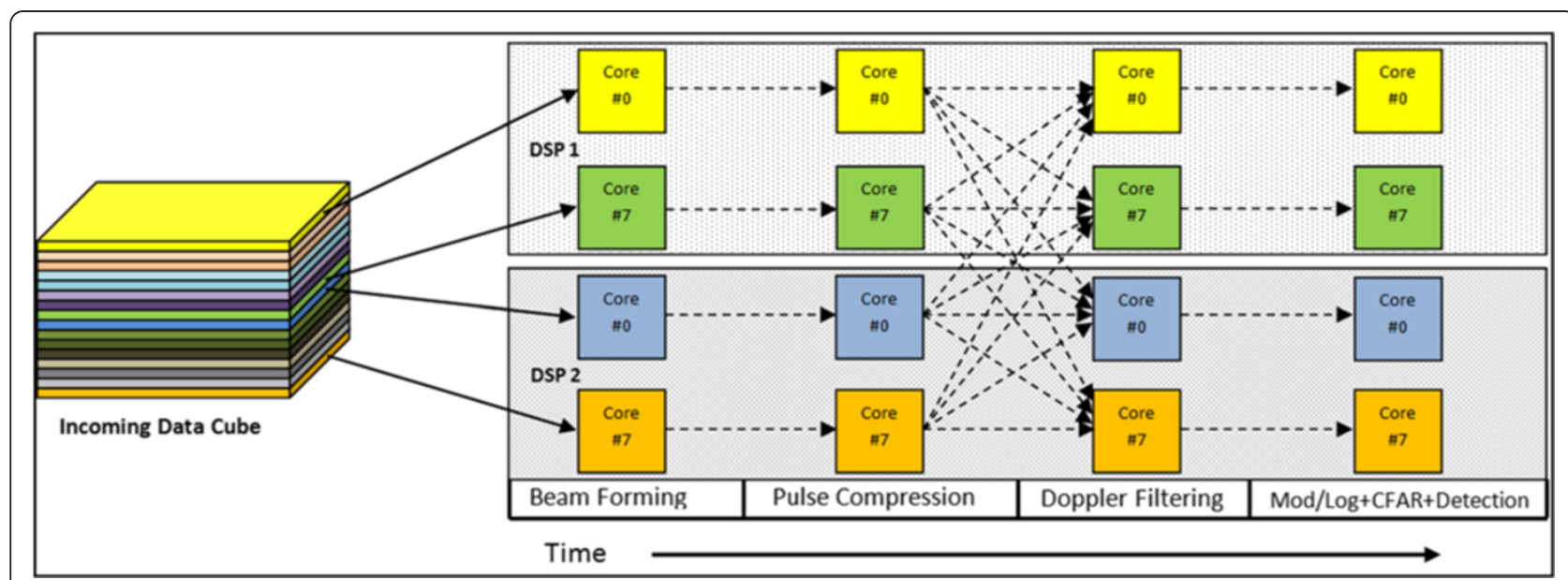

Figure 24 Straightforward model after optimizations.

\subsubsection{Optimization synthesis}

The optimizations that we have proposed to reduce interprocessor communication in the basic straightforward mapping model have completely eliminated inter-processor communication between the beamforming and pulse compression, as well as between the Doppler filtering and the post-processing (Figure 24). As a result, the processing of beamforming and pulse compression, as well as the processing of Doppler filtering and the post-processing can be fused in one single block (Figure 25). The proposed optimizations have also greatly reduced inter-processor communication between the pulse compression and Doppler filtering. The parallel machine executes $94 \%$ of inter-processor communication in parallel with the pulse compression.

The optimizations performed to the straightforward mapping model are based on distributing the beamforming and pulse compression on DSP cores along pulse dimension $(\mathrm{Np})$ and distributing the Doppler filtering and the postprocessing on DSP cores along beam dimension $(\mathrm{Nb})$. If these two parameters change and they are not equal or not multiple of the numbers of DSP cores, one or multiple DSP cores will process multiple beams or pulses or it will stay in idle. Changing of the other parameters of the Pulse-Doppler radar signal processing chain which are number of ranges $(\mathrm{Nr})$, number of channels $(\mathrm{Nc})$, and number of Doppler filters (Nd) does not influence on optimizations performed to the straightforward model.

\subsection{Experiments and results}

The implementation results of the Pulse-Doppler radar signal processing chain use case on the proposed parallel machine using the straightforward model before optimization

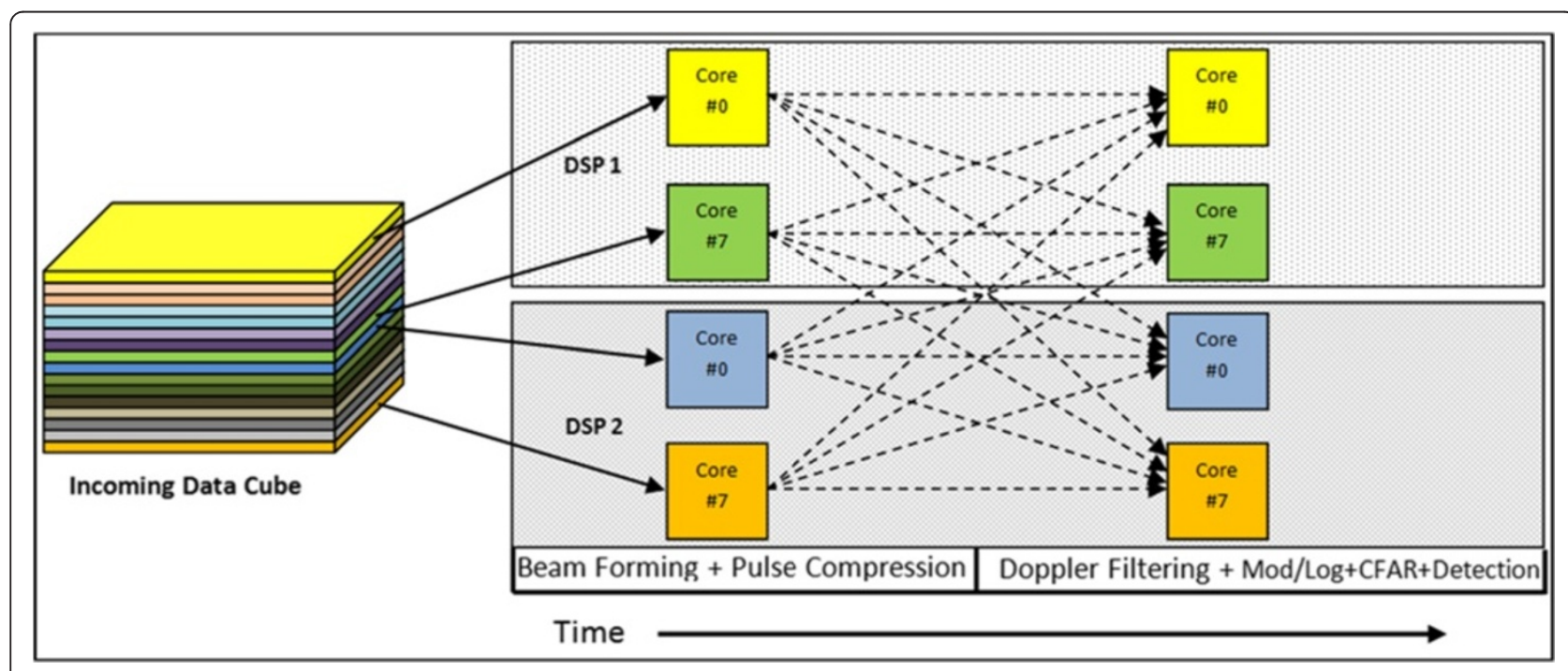

Figure 25 Straightforward model after fuse result. 


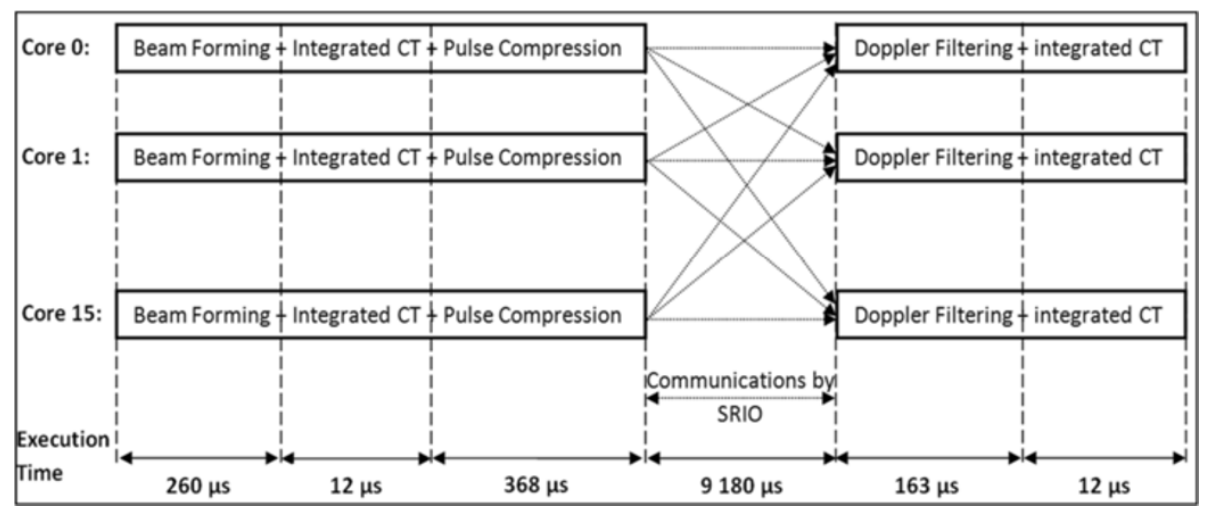

Figure 26 Implementation results before optimization.

are presented in Figure 26. The total processing time obtained is equal to $10 \mathrm{~ms}$. It is much greater than the realtime constraint of the use case which is about $1.64 \mathrm{~ms}$. The parallel efficiency is about 9\%. The inter-processor communication by SRIO takes $92 \%$ of the total processing time.

The implementation results using the optimized straightforward model are presented in Figure 27. The obtained total processing time is equal to $0.96 \mathrm{~ms}$ which is lower than the real-time constraint. The parallel efficiency obtained is about $90 \%$. Previous work [3,21-25] using the straightforward model to distribute a radar application on a parallel machine based on a RapidIO interconnect have achieved a parallel efficiency that does not exceed $40 \%$.

\section{Conclusions}

The Pulse-Doppler radars require high-processing power. A massively parallel machine was presented in this paper with the aim to implement the Pulse-Doppler radar's signal processing chain in real-time fashion. It was based on the C6678 multi-core DSP as the basic processing element and on SRIO as a high-performance inter-processor communication bus. We used a straightforward model to distribute the processing on the parallel machine. This model produces low latency but generates communication inefficiency which limits the performance of the system. The major contributions of this paper are to propose optimizations that reduce the inter-processor communication generated by the straightforward model in the corner turn stages and to improve the parallel efficiency of the system. A use case of Pulse-Doppler radar signal processing chain and an experimental platform of a parallel machine with 16 DSP cores interconnected by SRIO have been proposed to illustrate and validate the concept of the proposed mapping model.

The proposed optimizations have greatly reduced inter-processor communication in the parallel machine. Communications between the beamforming and pulse compression, as well as between the Doppler filtering and the post-processing have been completely eliminated. As a result, the processing of beamforming and pulse compression, as well as the processing of Doppler filtering and the post-processing has been fused in one single block. In addition, the parallel machine executes $94 \%$ of inter-processor communication between the pulse compression and Doppler filtering in parallel with the pulse compression.

Experimental results show that the proposed parallel machine and all optimizations performed to the straightforward

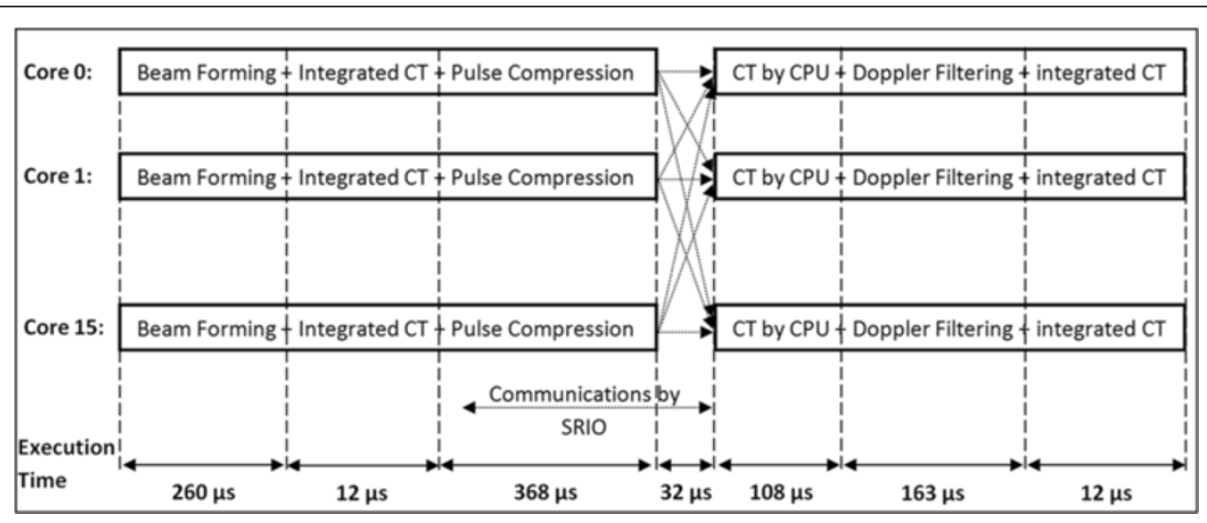

Figure 27 Implementation results after optimization. 
mapping model have allowed processing the Pulse-Doppler radar signal processing chain use case in real-time fashion. The parallel efficiency obtained is about $90 \%$, which is much greater than $40 \%$ found in the previous work.

\section{Competing interests}

The authors declare that they have no competing interests.

\section{Acknowledgements}

This research is sponsored in part by a research and development contract from Thales Air Systems and the Moroccan Foundation for Advanced Science, Innovation, and Research.

\section{Author details}

${ }^{1}$ Ecole Nationale des Sciences Appliquees - Marrakech, University of Cadi Ayyad, Marrakech, Morocco. ${ }^{2}$ Thales Air Systems, Paris, France. ${ }^{3}$ Moroccan Foundation for Advanced Science, Innovation and Research, Rabat, Morocco.

\section{Received: 13 June 2014 Accepted: 28 October 2014}

Published: 8 November 2014

\section{References}

1. DC Schleher, MTl and Pulsed Doppler Radar (Artech House, Inc, Nonwood, MA, 1991)

2. M Bahtat, S Belkouch, P Elleaume, P Le Gall, Efficient implementation scheme of a real-time radar beamformer on a VLIW DSP processor, TMS320C66x TI DSP implementation, in 2012 International Conference on Complex Systems (ICCS) (IEEE, Agadir, Morocco, 2012), pp. 1-6

3. D Bueno, C Conger, A Leko, I Troxel, AD George, Virtual prototyping and performance analysis of RapidIO-based system architectures for space-based radar, in Eighth Annual Workshop on High-Performance Embedded Computing (HPEC) (Massachusetts Institute of Technology Lincoln Laboratory, Lexington, MA, USA, 2004), pp. 28-30

4. TMS320C6678 Multicore Fixed and Floating-Point Digital Signal Processor (Texas Instruments, USA, 2014). http://www.ti.com/lit/ds/symlink/ tms320c6678.pdf

5. G Yang, JD Bakos, Sparse matrix-vector multiply on the Texas Instruments C6678 digital signal processor, in 2013 IEEE 24th International Conference on Application-Specific Systems, Architectures and Processors (ASAP) (IEEE, Washington, DC, USA, 2013), pp. 168-174

6. R Mego, T Fryza, Performance of parallel algorithms using OpenMP, in 2013 23rd International Conference on Radioelektronika (RADIOELEKTRONIKA) (IEEE, Pardubice, Czech Republic, 2013), pp. 236-239

7. Z ZhenHuan, $H$ Wei, $T$ Yan, Y DaWei, W XianHong, A design of versatile image processing platform based on the dual multi-core DSP and FPGA, in 2012 Fifth International Symposium on Computational Intelligence and Design (ISCID) (IEEE, Hangzhou, China, 2012), pp. 236-239

8. M Ali, E Stotzer, FD Igual, RA van de Geijn, Level-3 BLAS on the TI C6678 multi-core DSP, in 2012 IEEE 24th International Symposium on Computer Architecture and High Performance Computing (SBAC-PAD) (IEEE, New York, NY, USA, 2012), pp. 179-186

9. W Min, $S$ Xiu-qin, The design of high performance tracking system based on multi C6678, in 2012 5th International Congress on Image and Signal Processing (CISP) (IEEE, Chongqing, Sichuan, China, 2012), pp. 1348-1351

10. W Dan, M Ali, Synthetic aperture radar on low power multi-core digital signal processor, in 2012 IEEE Conference on High Performance Extreme Computing (HPEC) (IEEE, Waltham, MA, USA, 2012), pp. 1-6

11. G Chengfei, L Xiangyang, C Wenge, J Gaowei, T Haishan, Matrix transposition based on C6678, in 2012 5th Global Symposium on Millimeter Waves (GSMM) (IEEE, Harbin, Heilongjiang, China, 2012), pp. 29-32

12. A Klilou, S Belkouch, P Elleaume, P Le Gall, F Bourzeix, MM Hassani, Performance optimization of high-speed interconnect SRIO for onboard processing, in 2012 International Conference on Complex Systems (ICCS) (IEEE, Agadir, Morocco, 2012), pp. 1-6

13. RapidIO Interconnect Specification, LP-Serial Physical Layer Specification Rev. 2.1 (RapidlO Trade Association, Austin, TX, USA, 2009)

14. J Adams, C Katsinis, W Rosen, D Hecht, V Adams, HV Narravula, S Sukhtankar, R Lachenmaier, Simulation experiments of a high-performance RapidlO-based processing architecture, in IEEE International Symposium on Network Computing and Applications, 2001 (NCA 2001, 2001) (IEEE, Cambridge, MA, USA, 2001), pp. 336-339
15. X Zhang, G Liu, M Gao, A high-performance scalable computing system for real-time signal processing applications, in Congress on Image and Signal Processing, 2008 (CISP '08, 2008) (IEEE, Sanya, China, 2008), pp. 556-560

16. X Zhang, M Gao, G Liu, A scalable heterogeneous multi-processor signal processing system based on the RapidIO interconnect, in International Symposium on Intelligent Information Technology Application Workshops, 2008 (IITAW '08, 2008) (IEEE, Shanghai, China, 2008), pp. 761-764

17. W Changrui, C Fan, C Huizhi, A high-performance heterogeneous embedded signal processing system based on Serial RapidlO interconnection, in 2010 3rd IEEE International Conference on Computer Science and Information Technology (ICCSIT) (IEEE, Chengdu, China, 2010), pp. 611-614

18. Y Zhang, Y Wang, P Zhang, A high-performance scalable computing system on the RapidIO interconnect architecture, in 2010 International Conference on Cyber-Enabled Distributed Computing and Knowledge Discovery (CyberC) (IEEE, Huangshan, China, 2010), pp. 288-292

19. J Zhang, S Hb, W Q-Z, J Zhang, Research and implement of SRIO based on Mul-DSP, in Conference on Computational Intelligence and Software Engineering (IEEE, Wuhan, China, 2009), pp. 1-4

20. S Xue, J Wang, Y Li, Q Peng, Parallel FFT implementation based on multi-core DSPs, in 2011 International Conference on Computational Problem-Solving (ICCP) (IEEE, Chengdu, China, 2011), pp. 426-430

21. D Bueno, C Conger, A Leko, I Troxel, AD George, RapidlO-based space system architectures for synthetic aperture radar and ground moving target indicator, in Ninth Annual Workshop on High-Performance Embedded Computing (HPEC) (Massachusetts Institute of Technology Lincoln Laboratory, Lexington, MA, USA, 2005), pp. 20-22

22. D Bueno, A Leko, C Conger, I Troxel, AD George, Simulative analysis of the RapidIO embedded interconnect architecture for real-time, network-intensive applications, in 29th Annual IEEE International Conference on Local Computer Networks (LCN) via the IEEE Workshop on High-Speed Local Networks (HSLN) (IEEE, Tampa, Florida, USA, 2004), pp. 710-717

23. C Conger, D Bueno, AD George, Experimental analysis of multi-FPGA architectures over RapidlO for space-based radar processing, in Tenth Annual Workshop on High-Performance Embedded Computing (HPEC) (Massachusetts Institute of Technology Lincoln Laboratory, Lexington, MA, USA, 2006)

24. D Bueno, C Conger, AD George, I Troxel, A Leko, RapidlO for radar processing in advanced space systems. ACM Trans. Embed. Comput. Syst. 7(1), 1-38 (2007)

25. D Bueno, C Conger, AD George, Optimizing RapidIO architectures for onboard processing. ACM Trans. Embed. Comput. Syst. 9(3), 1-30 (2010)

26. G Schorcht, I Troxel, K Farhangian, P Unger, D Zinn, CK Mick, A George, H Salzwedel, System-level simulation modeling with MLDesigner, in 11th IEEE/ ACM International Symposium on Modeling, Analysis and Simulation of Computer Telecommunications Systems, 2003 (MASCOTS 2003, 2003) (IEEE, Orlando, FL, USA, 2003), pp. 207-212

27. E Brookner, Phased-array radar. Sci. Am. 252, 94-102 (1985)

28. BD Van Veen, KM Buckley, Beamforming: a versatile approach to spatial filtering. IEEE ASSP Mag. 5(2), 4-24 (1988)

29. PZ Peebles, Radar Principles (Wiley-India, 1998

30. F Bin Khalid, RA Amjad, MA Chohan, MM Khizar, FPGA based real-time signal processor for Pulse Doppler radar, in 2012 International Conference on Informatics, Electronics \& Vision (ICIEV) (IEEE, Dhaka, Bangladesh, 2012), pp. 362-366

31. H Izumi, K Sasaki, K Nakajima, H Sato, An efficient technique for corner-turn in SAR image reconstruction by improving cache access, in Proceedings International Symposium on Parallel and Distributed Processing (IPDPS 2002, 2002) (IEEE, Ft. Lauderdale, FL, USA, 2002), pp. 3-8

32. TMDXEVM6678L EVM Technical Reference Manual Version 2.0 (Texas Instruments, USA, 2011). http://wfcache.advantech.com/support/ TMDXEVM6678L_Technical_Reference_Manual_2V00.pdf

33. T Scheckel, Serial RapidIO: Benefiting system interconnects, in IEEE International SOC Conference, 2005 (IEEE, Herndon, VA, USA, 2005), pp. 317-318

34. KeyStone Architecture Serial RapidIO (SRIO) User Guide (Texas Instruments, USA, 2012). http://www.ti.com/lit/ug/sprugw1b/sprugw1b.pdf

35. S-RIO Development Platform Gen2 (Silicon Turnkey Express, USA, 2011). http://silicontkx.com/pdfs/SRDP2.pdf

doi:10.1186/1687-6180-2014-161

Cite this article as: Klilou et al:: Real-time parallel implementation of Pulse-Doppler radar signal processing chain on a massively parallel machine based on multi-core DSP and Serial RapidIO interconnect. EURASIP Journal on Advances in Signal Processing 2014 2014:161. 